\title{
Coenzyme $Q$ and the Respiratory Chain: Coenzyme Q Pool and Mitochondrial Supercomplexes
}

\author{
José Antonio Enriquez ${ }^{\mathrm{a}} \quad$ Giorgio Lenaz ${ }^{\mathrm{b}}$

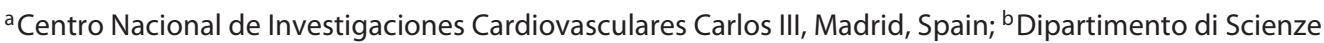 \\ Biomediche e Neuromotorie, Università di Bologna, Bologna, Italy
}

\author{
Key Words \\ Coenzyme Q · Mitochondria · Respirasome · Respiratory \\ chain - Respiratory complexes · Supercomplexes · \\ Ubiquinone
}

\begin{abstract}
Two alternative models of organization of the mitochondrial electron transport chain (mETC) have been alternatively favored or questioned by the accumulation evidences of different sources, the solid model or the random collision model. Both agree in the number of respiratory complexes (I-IV) that participate in the $\mathrm{mETC}$, but while the random collision model proposes that Complexes I-IV do not interact physically and that electrons are transferred between them by coenzyme $\mathrm{Q}$ and cytochrome $\mathrm{c}$, the solid model proposes that all complexes super-assemble in the so-called respirasome. Recently, the plasticity model has been developed to incorporate the solid and the random collision model as extreme situations of a dynamic organization, allowing super-assembly free movement of the respiratory complexes. In this review, we evaluate the supporting evidences of each model and the implications of the super-assembly in the physiological role of coenzyme Q.

(c) 2014 S. Karger AG, Basel
\end{abstract}

The classical electron transfer chain was described as the functional sequence of 4 major multi-subunit enzymatic complexes, randomly dispersed in the inner mitochondrial membrane and designated as $\mathrm{NADH}$-coenzyme Q (CoQ) reductase (Complex I, $\mathrm{C}_{\mathrm{I}}$ ), succinate-CoQ reductase (Complex II, $\mathrm{C}_{\mathrm{II}}$ ), ubiquinol-cytochrome $\mathrm{c}$ reductase (Complex III, $\mathrm{C}_{\mathrm{III}}$ ), and cytochrome c oxidase (Complex IV, $\mathrm{C}_{\mathrm{IV}}$ ); in this view, the enzyme complexes are connected by 2 mobile redox-active molecules, i.e. a lipophilic quinone, designated CoQ or ubiquinone, embedded in the membrane lipid bilayer, and a hydrophilic heme protein, cytochrome $c$, localized on the external surface of the inner membrane [Green and Tzagoloff, 1966].

The inner membrane contains other proteins with electron transfer activity in smaller amounts; among these there are electron transfer flavoproteins (ETF), capable of feeding electrons to the respiratory chain by pathways not involving Complex I and/or NAD, i.e. glycerol-3-phosphate dehydrogenase, ETF-ubiquinone oxidoreductase, dihydroorotate dehydrogenase, choline dehydrogenase, sulfide CoQ reductase (SQR), and proline dehydrogenase besides alternative NADH dehydrogenases in mitochondria from several organisms, especially plants and fungi. Moreover, alternative or branched

\section{KARGER}

E-Mail karger@karger.com

www.karger.com/msy
(C) 2014 S. Karger AG, Basel

$1661-8769 / 14 / 0054-0119 \$ 39.50 / 0$
José Antonio Enriquez

Centro Nacional de Investigaciones Cardivasculares Carlos III

Melchor Fernández Almagro 3

ES-28029 Madrid (Spain)

E-Mail jaenriquez@cnic.es 
pathways of electron transfer, differing from CoQ, also occur, e.g. alternative ubiquinol oxidases from bacteria and plant and fungi mitochondria [Lenaz and Genova, 2010].

The traditional description considers the 4 complexes originally described by Green and Tzagoloff [1966] to be the structural core of the respiratory chain. Subsequently, other accessory enzymes feeding electrons to the chain have been added. Nevertheless, a substantial difference exists between 3 of the original complexes $\left(\mathrm{C}_{\mathrm{I}}, \mathrm{C}_{\mathrm{III}}\right.$, and $\mathrm{C}_{\mathrm{IV}}$ ) and Complex II, since the latter shares some important properties with the accessory enzymes, i.e. they give electrons to CoQ without creation of a transmembrane proton gradient; in other words they are required for oxidative phosphorylation (OXPHOS) but do not participate directly in energy conservation. Moreover, contrary to the 'core' complexes, the accessory enzymes do not have subunits encoded by mitochondrial DNA. Furthermore, their supramolecular organization is probably different, since the 3 'core' complexes are associated together in a supramolecular assembly, whereas the remaining accessory enzymes appear to be free in a random organization.

The systematic resolution and reconstitution of the 4 respiratory complexes from mitochondria were accomplished by Hatefi et al. [1962a], leading Green and Tzagoloff [1966] to postulate that the overall respiratory activity is the result of both intra-complex electron transfer in solid state between redox components having fixed steric relation and inter-complex electron transfer ensured by rapid diffusion of the mobile components acting as co-substrates, i.e. CoQ and cytochrome c. This proposal was substantially confirmed over the following years, leading to the postulation by Hackenbrock et al. [1986] of the random collision model (RCM) of electron transfer. The organization of the respiratory chain has represented a major research subject in the 1970-80s, culminating with the acceptation of the RCM by the majority of scientists in the field.

Evidence in favor of the RCM derived from 3 major kinds of observations:

(a) The integral proteins of the inner membrane are randomly distributed in the bilayer, and phospholipid dilution of the mitochondrial membrane proteins slows down electron transfer [Schneider et al., 1982].

(b) Electron transfer in the CoQ and cytochrome $\mathrm{c}$ region obeys pool behavior according to the equation developed for CoQ by Kröger and Klingenberg [1973].

(c) Electron transfer follows saturation kinetics with respect to CoQ and cytochrome c concentrations [MacLennan et al., 1966; Estornell et al., 1992].
On the other hand, circumstantial evidence against a random distribution of respiratory complexes came from early investigations reporting isolation of Complex I-III [Hatefi et al., 1962b] and Complex II-III units [Yu et al., 1974], indicating that such units may be preferentially associated in the native membrane. The view of preferential associations between complexes was never abandoned, but only a minority of scientists was favoring such a possibility [Lenaz and Genova, 2010].

Much more recently, new evidence of multi-complex units in yeast and mammalian mitochondria has been obtained introducing blue native polyacrylamide gel electrophoresis (BN-PAGE) [Schägger and Pfeiffer, 2000]. In mitochondria of bovine heart, Complex I-III interactions were apparent from the presence of supercomplexes also comprising either Complex III alone or both Complexes III and IV at different copy numbers (respirasomes), and only $14-16 \%$ of total Complex I was found in free form in the presence of digitonin [Schägger and Pfeiffer, 2001]; so it seems likely that all Complex I is bound to Complex III in physiological conditions in the absence of detergents. On the other hand, knowing the accurate stoichiometry of oxidative phosphorylation complexes, it is plausible that approximately $30 \%$ of total Complex III in bovine mitochondria is not bound to monomeric Complex I. The fraction of Complex IV in free form represents $>85 \%$ of total cytochrome oxidase of mitochondria. Since these initial studies, respiratory supercomplexes have been identified in mitochondria from many different species [Wittig and Schägger, 2008; Lenaz and Genova, 2012].

Associations of Complex II with other complexes of the OXPHOS system could not be identified in most of the studies. However, monomeric to trimeric forms of Complex II [Sousa et al., 2011] and potential incorporation in superstructures [Acín-Peréz et al., 2008; Kulawiak et al., 2013] have been reported, representing, in any case, a minor proportion of the overall mitochondrial Complex II. In addition, no evidence for supercomplex association has been found for other ancillary enzymes feeding electrons to CoQ; thus the supercomplex organization appears to be a prerogative of the energy-conserving respiratory complexes. However, it has been suggested that a multifunctional fatty acid $\beta$-oxidation complex is formed within mitochondria that is physically associated with OXPHOS supercomplexes and promotes metabolic channeling of electrons directly from fatty acid $\beta$-oxidation to the respiratory chain [Wang et al., 2010]. 


\section{Molecular Structure of Supercomplexes}

Partial elucidation of the interaction of the individual respiratory complexes within the supercomplex was achieved by single-particle electron microscopy.

Dudkina et al. [2005] first presented the 2D electron projection maps of a plant supercomplex consisting of Complex I and dimeric Complex III ( $\left.\mathrm{III}_{2}\right)$. Class averages in single-particle processing showed characteristic Fshaped side views of the particle volume and roughly triangular top views.

Shortly later, Schäfer et al. [2006, 2007] provided initial insights into the shape and size of 2 respiratory chain supercomplexes $\left(\mathrm{I}_{1} \mathrm{III}_{2}\right.$ and $\left.\mathrm{I}_{1} \mathrm{III}_{2} \mathrm{IV}_{1}\right)$ isolated from mitochondria of bovine heart, and they also generated 3D maps by random conical tilt electron microscopy analysis of the negatively stained multicomplex assemblies. The dimensions of the supercomplex in the membrane plane are 28 by $24 \mathrm{~nm}$. On the basis of the structural information gained by the 3D maps, they could propose a model of the spatial organization and mutual arrangement of all partner enzymes, showing extensive interaction of Complex III with the membrane arm of Complex I, while Complex IV, when present, interacts with the distal portion of the same arm. Furthermore, on the same basis, the ubiquinone and cytochrome $\mathrm{c}$ binding sites of each complex in the supercomplex $\mathrm{I}_{1} \mathrm{III}_{2} \mathrm{IV}_{1}$ appeared to be in proximity to the binding site of the succeeding complex in the respiratory chain, thus supporting the idea that direct substrate channeling occurs in the supercomplex with short diffusion distances for the mobile electron carriers.

Recently, new 3D maps at nanoscale resolution allowed interpretation of the architecture of mammalian respirasomes at the level of the secondary structure. Interestingly, in the new model by Dudkina et al. [2011], the single Complexes I, $\mathrm{III}_{2}$, and IV appear to be at some distance, suggesting that there is little close contact. In particular, the section through the model on the level of the membrane demonstrates gaps between Complexes $\mathrm{III}_{2}$ and IV within the membrane, whereas the same 2 complexes appear to contact each other in their matrix portions close to the membrane.

Althoff et al. [2011] also proposed that only few points of direct contact are allowed between the 3 complexes in the mammalian supercomplex $\mathrm{I}_{1} \mathrm{III}_{2} \mathrm{IV}_{1}$, because average distances exceed $2 \mathrm{~nm}$. Moreover, at $19-\AA$ r resolution the membrane-embedded part of the supercomplex shows intermediate values of density between that of soluble protein and the hydrophobic membrane interior, sug-

CoQ Pool and Mitochondrial

Supercomplexes gesting that the supercomplex is held together at least partly by lipid-protein interactions. Likely, a gap filled with membrane lipid would also facilitate the diffusion of ubiquinol between Complex I and Complex III, and it is interesting to note that the CoQ-binding sites in Althoff s model (fig. 1) are conveniently placed for the efficient electron transfer over a short distance of about $13 \mathrm{~nm}$ between Complex I and the proximal Complex III monomer. It is proposed that the proximal monomer may be more effective in ubiquinol oxidation, while the distal one may be needed to transfer the electrons to cytochrome $\mathrm{c}$ via its flexible Rieske domain in accordance with the halfof-the-sites reactivity model of Complex III [Castellani et al., 2010]. Furthermore, a trajectory of $10 \mathrm{~nm}$ is envisaged in the respirasome along which cytochrome $\mathrm{c}$ may travel to shuttle electrons towards Complex IV (fig. 1).

It has to be kept in mind that, besides the lack of a crystal structure of the mammalian Complex I, several uncertainties are still present in the interpretation of the $3 \mathrm{D}$ maps: for example, in the maps there are additional density areas that cannot be filled by the current structural models of Complexes III and IV, suggesting that additional proteins may participate in the supercomplexes. This is the case of SCAFI, a recently reported protein required for the super-assembly of Complexes III and IV that is not present in the free complexes [Lapuente-Brun et al., 2013] or the reassignment of NDUF4A as a structural subunit of Complex IV which is not present in the $\mathrm{X}$-ray structure [Balsa et al., 2012].

\section{Stability of Supercomplexes: Role of Phospholipids}

All purified preparations of mitochondrial electron transfer enzymes are isolated as lipoprotein complexes, the extent of associated lipid depending upon the particular method used for isolation and the phospholipid composition reflecting that for the mitochondrial inner membrane. Predominant phospholipids include cardiolipin, phosphatidylcholine, phosphatidylethanolamine, and lesser amounts of neutral lipids and phosphatidylinositol. A dispersive solubilization effect and a catalytic effect, which can be specifically fulfilled only by cardiolipin, are 2 of the distinguished roles for such membrane lipids. In addition, they can participate in the molecular linkage between the respiratory complexes and, particularly in the case of Complex I and Complex III, can provide a sufficiently lipophilic environment for the interaction of the lipophilic electron carrier ubiquinone [Lenaz and Genova, 2007]. 


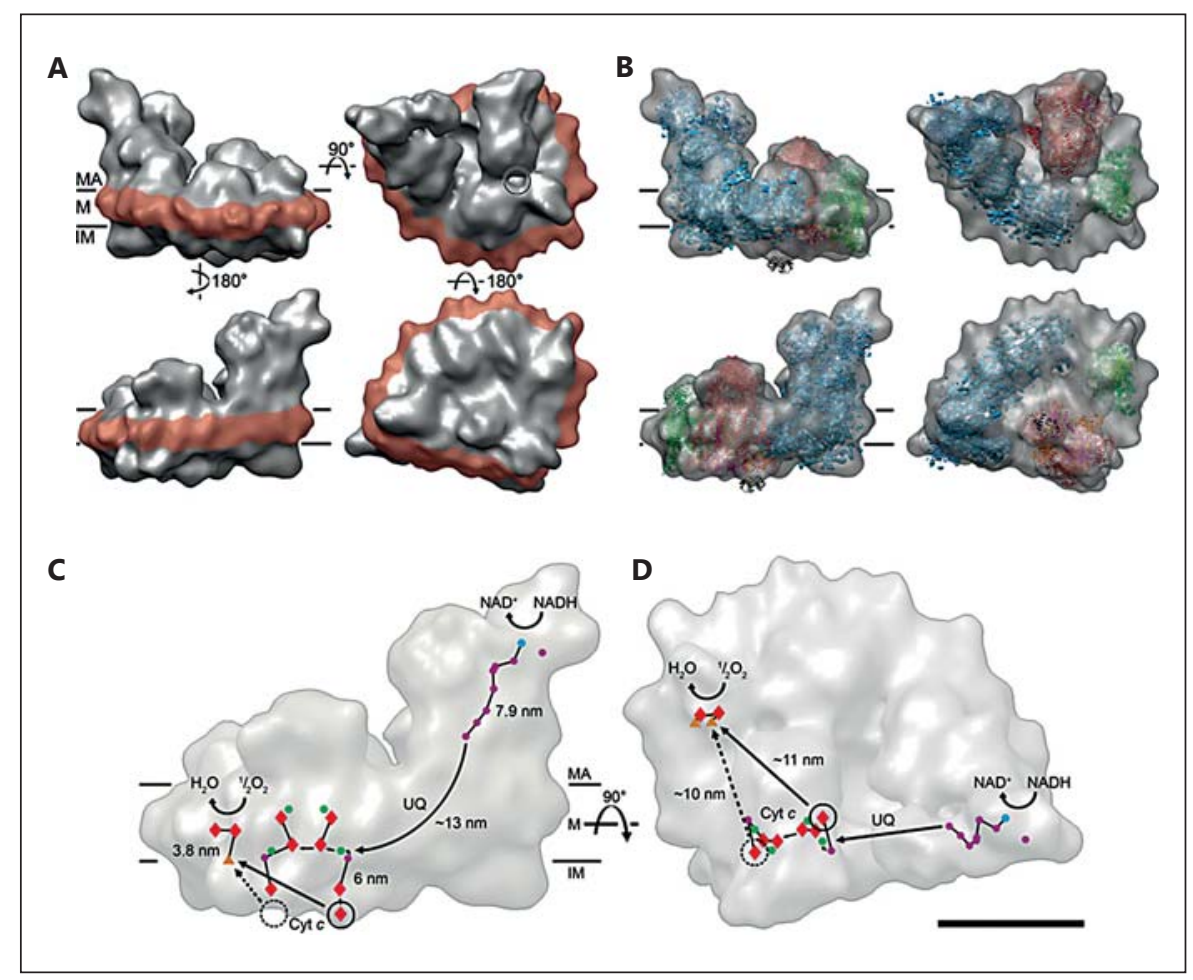

Fig. 1. A, B Respirasome 3D map and fitted X-ray structures. C, D Electron transfer pathways in the supercomplex. A Cryo-EM 3D map as seen from the 2 opposite sides (left), from the matrix (top right), and the intermembrane space (lower right). The amphipol belt is shown in red. The circle marks the gap between Complex I and Complex III. B X-ray structures of component complexes $($ blue $=$ Complex I, red $=$ Complex III, green $=$ Complex IV $)$ and cytochrome c (black) fitted to the 3D cryo-EM map. C, D Outline of the supercomplex with cofactors active in electron transport, FMN (blue circles), iron-sulphur clusters (purple circles), quinols/ stigmatellins (green circles), hemes (red diamonds), and copper atoms (orange triangles), seen from the membrane $(\mathbf{C})$ and from the matrix (D). Electron trajectories are marked in black. The dashed circle marks the distal cytochrome $\mathrm{c}$ binding site which is unoccupied in the supercomplex. Straight arrows in C and D indicate the shortest distances from the cytochrome $\mathrm{c}$ binding sites on Complex III to the site of cytochrome c oxidation in Complex IV. The shorter proximal branch may be preferred for electron transport. $\mathrm{MA}=$ Matrix; $\mathrm{M}=$ membrane; $\mathrm{IM}=$ intermembrane space; $\mathrm{UQ}=$ ubiquinol; Cyt $c=$ cytochrome $\mathrm{c}$. Scale bar $=10 \mathrm{~nm}$. Taken from Althoff et al. [2011] with small modifications.
The forces responsible for supercomplex association appear to strongly depend on the lipid content and composition and likely the shape of the inner mitochondrial membrane [Lenaz and Genova, 2007]. In particular, previous studies from our laboratory have shown that reconstitution of a binary I/III mitochondrial protein fraction at a high lipid to protein ratio (>10:1) prevents formation of the I-III supercomplex as shown by BN-PAGE and kinetic studies [Genova et al., 2008]. In other words, it appears that dilution of the proteins with an excess of phospholipids may weaken the forces holding together the respiratory complexes.

Among lipids, cardiolipin and phosphatidylethanolamine are crucial for mitochondrial functions; both are non-bilayer-forming phospholipids due to their small polar heads compared with the bulky non-polar tails [van den Brink-van der Laan et al., 2004; Osman et al., 2011].

Cardiolipin, the signature phospholipid of mitochondria, is a lipid dimer that is required for a diverse range of mitochondrial activities beyond the process of ATP production. Thus, derangements in cardiolipin metabolism lead to pathological conditions. Cardiolipin is synthesized in mitochondria; after its biosynthesis, the acyl chain composition of cardiolipin is modified by 3 distinct remodeling enzymes that produce the tissue-specific mature form of cardiolipin. Mutations of these enzymes produce modified molecules that have been linked to mitochondrial dysfunction [Claypool and Koehler, 2012].

The phospholipids in closest vicinity to the protein surface, as well as those in the free bilayer, are highly mo- 
bile and free to exchange, but cardiolipin is tightly bound, being more likely buried within the protein complexes [Kang et al., 1979; Lange et al., 2001; Sedlák and Robinson, 1999]. The absolute requirement of cardiolipin for the activity of cytochrome oxidase, Complex I, and Complex III suggests that this phospholipid plays a crucial role in the coupled electron transfer process [Fry and Green, 1980, 1981].

There are now extensive indications that cardiolipin stabilizes respiratory supercomplexes as well as the individual complexes. Evidence for this function has been largely collected in yeast mutants.

In a yeast mutant lacking cardiolipin, the $\mathrm{III}_{2} \mathrm{IV}_{2}$ supercomplex was significantly less stable than supercomplexes in the parental strain. Other phospholipids that are increased in the mutant, including phosphatidylethanolamine and phosphatidylglycerol, could not substitute for cardiolipin and could not prevent dissociation of supercomplexes [Zhang et al., 2002; Pfeiffer, 2003]. The putative direct protein-protein interaction of cytochrome oxidase and Complex III in yeast is proposed to involve also one molecule of cardiolipin and one of phosphatidylethanolamine tightly bound in a cavity of the membrane-imbedded domain of Complex III [Lange et al., 2001], suggesting that the 2 phospholipids can provide a flexible linkage between the interacting subunits of Complexes III and IV, as well as with the ADP/ATP carrier that is also known to exist in physical association with the III-IV supercomplex [Claypool et al., 2008]. Site-directed mutagenesis investigations on specific cardiolipin interaction for supercomplex formation in the cytochrome $\mathrm{bc}_{1} \mathrm{com}$ plex [Wenz et al., 2009] indicated that cardiolipin stabilizes supercomplex formation by neutralizing the charges of lysine residues in the interaction domain of Complex III with cytochrome oxidase.

In addition, the stability and assembly of Complex IV were found to be reduced in yeast cells lacking Taz1 [Brandner et al., 2005], the orthologous of human Tafazzin, an acyl transferase involved in the synthesis of mature tetralinoleyl cardiolipin [Neuwald, 1997]. Mutations of Tafazzin (approved symbol: TAZ) in humans result in Barth syndrome, a cardio-skeletal myopathy with neutropenia, characterized by respiratory chain dysfunction. Significantly, McKenzie et al. [2006] found that the cardiolipin defect in Barth syndrome results in destabilization of the supercomplexes by weakening the interactions between the respiratory complexes.

It is well documented that exposure of mitochondria to reactive oxygen species (ROS) can affect the respiratory activity via oxidative damage of cardiolipin which is required for the optimal functioning of the enzyme complexes [Paradies et al., 2000, 2002, 2010; Petrosillo et al., 2003]. Genova et al. [2008] demonstrated by flux control analysis that the maintenance of a I-III supercomplex after reconstitution of a protein fraction enriched with Complex I and III $\left(\mathrm{R}_{4} \mathrm{~B}\right)$ into phospholipid vesicles at high protein to lipid ratios (see above) is abolished if lipid peroxidation is induced by $2,2^{\prime}$-azobis-(2-amidinopropane) dihydrochloride (AAPH) before reconstitution. Evidently, the distortion of the lipid bilayer induced by peroxidation and the alteration of the tightly bound phospholipids determine dissociation of the supercomplex originally present in the lipid-poor preparation.

In contrast with cardiolipin depletion that destabilizes supercomplexes, a recent study reported that phosphatidylethanolamine depletion tends to favor the formation of larger supercomplexes between Complex III and IV in mitochondria of Saccharomyces cerevisiae [Böttinger et al., 2012]. The reason why cardiolipin and phosphatidylethanolamine, both non-bilayer-forming phospholipids, behave in an opposite way on supercomplex stability has been ascribed to their different charge, the former being an anionic phospholipid and the latter zwitterionic [Böttinger et al., 2012].

\section{Coexistence of Fluid Structure and Supercomplexes: The Plasticity Model}

The organization of the mitochondrial respiratory chain as supercomplexes has been sustained on structural evidence at first, the co-migration of respiratory complexes either in BN-PAGE or in sucrose gradients and by electron microscopy structural analysis upon purification. Despite that, 2 fundamental assumptions have been established to propose a radical change in our understanding of the structural organization of the mitochondrial electron transport chain. First, the RCM has to be discarded in favor of a model in which respiratory complexes are organized in respirasomes (containing Complex I, III, and IV; solid model). Second, all the other structures observed in the purification protocols should be considered breaking pieces of the original respirasome. None of these methodologies, however, have been able to provide experimental support to sustain these critical assumptions. Very relevant, they have been unable to determine if the supercomplexes containing Complexes I, III, and IV (the so-called respirasome) were able to respire. Without solving this, even if accepted as real entities, supercomplexes could have roles different from these 
Fig. 2. Plasticity model of the mitochondrial OXPHOS system organization. Schematic representation of the 'classical' fluid (A) and solid (B) models for the organization of the OXPHOS system and a 'plasticity model' (C). The shape and color code for representing the individual complexes can be seen in $\mathbf{A}$; coenzyme $\mathrm{Q}$ is represented as small red stars and cytochrome $\mathrm{c}$ as purple triangles. For supercomplexes in C, only one complex unit of each type is represented in the different supercomplex associations, although the actual stoichiometry may vary. Image is taken from Acín-Peréz et al. [2008].

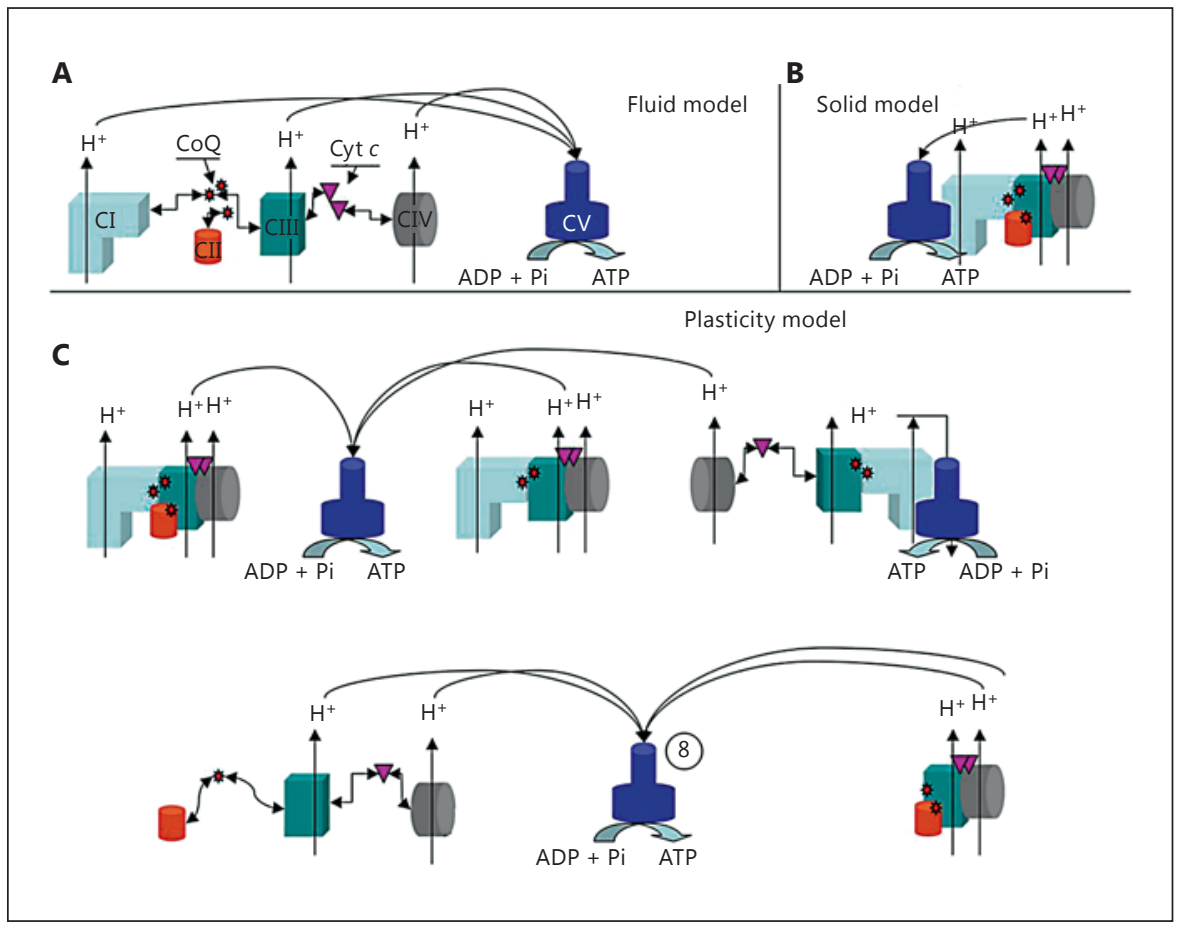

of respiration (i.e. storage of yet inactive respiratory complexes, structural organizers of the inner membrane, etc.). It was demonstrated that purified respirasomes were able to respire in a Clark's electrode [Acín-Peréz et al., 2008], and it was established that other types of associations than I+III or III+IV were also formed, regardless of the presence of the third partner of the respirasome, strongly suggesting that they can also be present in vivo [Acín-Peréz et al., 2008]. Therefore, we reasoned that the variety of associations between respiratory complexes was larger than the respirasome and that the free complexes likely co-exist with supercomplexes. In this context we proposed an integrated model, the plasticity model, for the organization of the mitochondrial electron transport chain. The previous opposed models, solid versus fluid, would be 2 extremely allowed and functional situations of a dynamic range of molecular associations between respiratory complexes [Acín-Peréz et al., 2008]. The stoichiometry of the complexes and the variable stability of different free versus associated structures under different physiological conditions would allow a landscape of structural options (fig. 2).

The proportion of the different ratios between complexes and supercomplexes is cell type specific and responds to physiological stimuli (see below). A typical fibroblast in the usual culture conditions ( $21 \%$ oxygen, high glucose) would favor: (a) 3 major flavors for Complex IV, free $(\sim 80 \%)$, associated with Complexes I and III in respirasomes $(\sim 15 \%)$ or associated only with Complex III ( 5\%); (b) 4 major flavors for Complex III, free, Complex I+III or interacting with Complex IV in the form of III+IV or I+III+IV; (c) 2 major flavors for Complex I (I+III or I+III+IV). Free Complex I was virtually absent, because it is rapidly degraded in the absence of Complex III [Acín-Peréz et al., 2004] or Complex IV [Diaz et al., 2005; Vempati et al., 2008].

Several in vitro studies support the idea that electron transfer in the respiratory chain can occur in the absence of supercomplexes. A fundamental prediction of the plasticity model is that in vivo the mitochondrial respiratory chain should be able to work when the formation of supercomplexes is prevented. Recently, a protein factor (SCAFI) has been described that is required to allow the integration of Complex IV into supercomplexes, either I+III or I+III+IV. Mice missing functional alternative oxidases (AOX) were also lacking supercomplexes containing Complex IV and therefore the respirasome. These animals contain supercomplexes I+III and are, however, healthy and fertile [Lapuente-Brun et al., 2013]. A more extreme situation can be achieved by the selective elimination of an individual respiratory complex and substituting it with an alternative electron acceptor/donor. This 
possibility is already present in nature, since the mitochondrial electron transport chain is branched in plants, fungi, yeast, and lower metazoans. Thus, AOX are able to oxidize CoQ to water, supplanting in that way the combined role of Complex III, cytochrome c, and Complex IV. Under these conditions, Complex I works perfectly in combination with AOX and in the absence of Complexes III and IV [Maas et al., 2009]. Expression of functional AOX in mammalian cells is feasible [Perales-Clemente et al., 2008], and it stabilizes Complex I and allows respiration in combination with AOX in the absence of Complex III or Complex IV [Guaras and Enriquez, unpubl. data]. Therefore, in vivo Complex I-dependent respiration does not require supercomplex formation. This demonstrates that the respiratory complexes can work either as free entities or as associated entities. What could then be the functional role for these associations?

Some observations in the literature indirectly support the view that the supercomplex organization may not be fixed but be in equilibrium with randomly dispersed complexes in living cells under physiological conditions. Besides phospholipid composition that may change by genetic or dietary reasons but involves a relatively long time-scale, some biochemical parameters at a shorter time-scale have been suggested to affect the supramolecular structure of the respiratory complexes. These are the mitochondrial membrane potential and the phosphorylation state of the protein subunits of the complexes.

Nevertheless, a recent study [Muster et al., 2010] suggests that supercomplex dissociation, at least in a random fashion, may not be a fast event. By fusing cells containing mitochondria with respiratory complexes labeled with different fluorescent proteins and resolving their timedependent re-localization in living cells, the authors found that a complete reshuffling of respiratory complexes throughout the entire chondriome in single HeLa cells occurs within $2-3 \mathrm{~h}$ by organelle fusion and fission. Moreover, polykaryons of fused cells completely re-mixed their complexes in $10-24 \mathrm{~h}$ in a progressive way. Nevertheless, the distribution of respiratory complexes and ATP synthase in fused hybrid mitochondria is not homogeneous but patterned: in co-expressing cells, Complex II is more homogeneously distributed than Complexes I and $\mathrm{V}$, arguing for its higher mobility and less integration in supercomplexes. Being these experiments the very first attempt to follow the temporal dynamic of respiratory complexes in situ, they are still very limited because the cristae and individual complexes or supercomplexes could not be discriminated by fluorescent microscopy. In addition, in order to minimize the interference in func-

CoQ Pool and Mitochondrial

Supercomplexes tion or structure, subunits of less pronounced functional importance and peripheral to the complex structure were chosen for labeling [Muster et al., 2010], and it could not be addressed how much of the labeled protein was in fact assembled into the final complexes and how much remained in sub-assembles. This is especially relevant, because in some cases (i.e. the labeled $30-\mathrm{kDa}$ Complex I protein), the sub-assembles could be either part of membrane-associated or matrix-associated subcomplexes [Dieteren et al., 2008], the diffusion properties of which would be very different.

\section{Membrane Potential}

The study of the influence of the mitochondrial transmembrane potential $\left(\Delta \mu_{\mathrm{H}+}\right)$ on the flux control exerted by cytochrome c oxidase on the respiratory activity in intact cells and isolated mitochondria indicated that, under conditions mimicking the mitochondrial state 4 respiration, the control strength of the oxidase is decreased in respect to endogenous state 3 respiration [Piccoli et al., 2006]. More recently, the extension of these studies to the other proton-translocating respiratory complexes (I and III) reveals that under conditions of low potential the sum of the flux control coefficients exceeded 1 , whereas at high potential the coefficients were much lower [Quarato et al., 2011]. Although the interpretation of the results in such a complex system is very difficult, the authors suggested that such a change in control strength might be featured in terms of an equilibrium between different organizational structures of the enzymatic complexes constituting the mitochondrial OXPHOS. In particular, the results were compatible with a random organization of cytochrome oxidase with respect to other complexes at high membrane potential (state 4) and with a respirasome organization at lower membrane potential (state 3); since the respiratory rate was high in state 3 conditions, the supercomplex organization would produce an extra advantage by raising the rate by channeling. We extended the study in actively phosphorylating human hepatoma HepG2 cells under conditions in which the electrical $(\Delta \Psi)$ and chemical $(\Delta \mathrm{pH})$ components of $\Delta \mu_{\mathrm{H}+}$ were selectively modulated by addition of ionophores. We concluded that $\Delta \Psi$ is predominantly responsible for the tight control exerted by cytochrome $c$ oxidase over endogenous respiration, whereas $\Delta \mathrm{pH}$ seems irrelevant in this respect [Dalmonte et al., 2009]. Although the molecular mechanisms leading to the assembly/disassembly of the supercomplexes have not been defined yet, it is not inconceivable that, given the membrane-integrated nature of the single complexes, electrostatic/hydrophobic interac- 
tions may enter into play in response to $\Delta \mu_{\mathrm{H}+}$. In this respect, recent investigations have revealed a surprising interplay between $\mathrm{pH}$ gradients, lipid composition and packing, and membrane cristae shape [Khalifat et al., 2008, 2011].

In potato tuber mitochondria, it was found that hypoxia dissociates the supercomplex I-III - IV into individual Complex I plus Complex III-IV units [Ramirez-Aguilar et al., 2011]; it was shown that the change was due to the acidification of the medium in hypoxia and that acidification could reproduce the same effect; moreover, the effect was not due to changes in the membrane potential.

\section{Posttranslational Changes}

It has been established that some of the mitochondrial complexes are subjected to reversible phosphorylation and dephosphorylation [Acín-Peréz et al., 2011a, b; Gong et al., 2012]. Mitochondria contain protein kinases and phosphatases, and both serine/threonine phosphorylation [Deng et al., 2011; Grimsrud et al., 2012] and tyrosine phosphorylation [Deng et al., 2011; Hébert Chatelain, 2013] of mitochondrial proteins are important in regulation of activity of these organelles.

Phosphorylation of Complex I has been shown to modify the activity of the enzyme and its ROS-generating capacity [Raha et al., 2002; Maj et al., 2004; Bellomo et al., 2006; Iuso et al., 2006; Papa et al., 2008]. Cyclic AMPdependent phosphorylation of the $18-\mathrm{kDa}$ subunit of Complex I, encoded by the nuclear NDUFS4 gene, is required for import of the subunit. Modulation of subunit phosphorylation by intramitochondrial protein kinase A and phosphoprotein phosphatase contributes to the stability of Complex I and stimulation of its activity [de Rasmo et al., 2008]. It is tempting to speculate that the increase of activity of Complex I and the decrease of ROS generation by phosphorylation may be, in part, the result of an enhanced stability of the I-III supercomplex; however, no studies to date have established any correlation between them. Phosphorylation of structural subunits of Complex III has also been reported, although its functional role remains undetermined [Zhao et al., 2011].

Lee et al. [2005] examined the cAMP-dependent phosphorylation of mitochondrial Complex IV isolated from fresh bovine liver and heart in the presence of theophylline, a phosphodiesterase inhibitor that induces high cellular cAMP levels. Under the conditions applied, they were able to demonstrate that phosphorylation of Tyr304 in subunit I (COX-I) leads to a strong decrease of $\mathrm{V}_{\max }$ in the isolated enzyme while increasing the $\mathrm{K}_{\mathrm{m}}$ for cytochrome c, so the residual oxidase activity is $<20 \%$ at substrate concentrations in the range of the physiological values, compared with saturating substrate concentration. The same effect on cytochrome oxidase kinetics was observed in a cell culture system after treatments that would increase protein phosphorylation (e.g. elevation of cAMP levels by glucagon addition or by forskolin activation of adenylyl cyclase). Complex IV is a target of the intramitochondrial cAMP-PKA signaling pathway [Acín-Peréz et al., 2009a]. Complex IV activity increases with the rise in mitochondrial matrix cAMP by cell-permeant cAMP analogs or by activating the soluble matrix adenylyl cyclase. The inhibition of PKA both in cell culture and in isolated mitochondria resulted in a decreased Complex IV activity [Acín-Peréz et al., 2009a]. Mitochondrial PKA-dependent phosphorylation of Complex IV was down-regulated in both cell culture and animal models of cytochrome $c$ oxidase (COX) deficiency. This promoted a ROS-dependent increase in mitochondrial biogenesis as a compensatory mechanism [Acín-Peréz et al., 2009b]. The expression of mitochondrial-targeted soluble adenylyl cyclase, which increases mitochondrial matrix cAMP, restored mitochondrial function and normalized ROS production and mitochondrial biogenesis in COX-deficient cells. This study represents the first example of a direct positive modulation of COX with clear implications for plausible therapeutic treatments of mitochondrial dysfunction [Acín-Peréz et al., 2009b]. Phosphorylation in Complex IV subunits was reported by mass spectrometry analysis [Helling et al., 2008]. The amino acid residue S58 in COXIV-1 (same as S34 published by Helling et al. [2008], the difference in the number being whether the signaling peptide is included or not) was described to be involved in the metabolic regulation of Complex IV [Acín-Peréz et al., 2011a]. A S58 COXIV-1 phosphorylation was proposed to have a dual function by promoting COXIV-1 phosphorylation coupled to prevention of COX allosteric inhibition by ATP. This mechanism would allow the cells to rapidly adapt to changes in substrate use and substrate storage [AcínPeréz et al., 2011a].

Stable phosphorylation sites in Complex IV are also detectable at tyrosine, serine, and threonine in subunit II and III, as well as at specific amino acid residues in subunits IV, Vab, VIabc, VIIabc, and VIII [Helling et al., 2008] whose function could be to change the binding affinity of Complex IV to specific proteins (i.e. binding of EGFR-pY845 to subunit II, viral protein HBx to subunit III, PKCe to subunit IV, NO synthase to subunit Va, subunit RIa of PKA to subunit $\mathrm{Vb}$, and androgen receptor to subunit $\mathrm{Vb}$ ). 
It is tempting to speculate that endocrine alterations may affect the assembly of Complex IV by hyper- or hypophosphorylation of some subunits in the complex. Indeed, cAMP- and PKA-dependent phosphorylation of Complex IV in heart mitochondria [Rosca et al., 2011] was found to be higher in free Complex IV than in the enzyme associated in a supercomplex, suggesting that phosphorylation prevents supercomplex association. Nevertheless, caution must be exerted when interpreting the role of posttranslational changes in supercomplex formation, since no causal correlation has yet been established.

\section{The Coenzyme Q Pool}

The notion of the CoQ pool as the mechanism for integrated electron transfer from dehydrogenases to cytochromes, described by the hyperbolic relationship between the observed rate of electron transfer of the entire respiratory chain and the rate of either reduction or oxidation of CoQ [Kröger and Klingenberg, 1973], has been widely accepted and is covered in all biochemistry textbooks.

Here, 2 separate points are discussed regarding this purpose. First, is the discovery of supercomplexes compatible with the existence of a pool of free CoQ molecules in the inner membrane? And second, if such pool exists, does it have a specific function in electron transfer?

There is no doubt that a mobile pool of CoQ coexists with protein-bound $\mathrm{CoQ}$. Is this pool just a reservoir of an excess of CoQ molecules without a specific function, or is the pool necessary for functioning of the respiratory chain and/or for additional functions?

The extent to which CoQ is bound to mitochondrial proteins is an important parameter in relation to its function. If we consider bound CoQ in a 1:1 stoichiometry with the complexes interacting with the quinone $\left(\mathrm{C}_{\mathrm{I}}, \mathrm{C}_{\mathrm{II}}\right.$, $\mathrm{C}_{\text {III }}$ ) in mitochondria of bovine heart, we come up to about $0.5 \mathrm{nmol} / \mathrm{mg}$ protein that would increase to ca. 0.8 nmol, assuming more than 1 site to be fully occupied in Complex I and Complex III. Since the total CoQ content is $>3 \mathrm{nmol} / \mathrm{mg}$ [Lenaz, 2001], we must assume that most CoQ $(>75 \%)$ is free in the bilayer. A direct study of the amount of CoQ bound to mitochondrial proteins in 5 different mammalian species showed that the protein-bound aliquot ranges between $10-32 \%$ of total CoQ [Lass and Sohal, 1999].

The CoQ pool is required for electron transfer from Complex II to Complex III; indeed Complex II kineti- cally follows pool behavior in mitochondria after extraction and reconstitution [Kröger and Klingenberg, 1973] with CoQ [Stoner, 1984] in accordance with the lack of supercomplexes found by both BN-PAGE and flux control analysis (see previous section). Furthermore, other enzymes such as glycerol-3-phosphate dehydrogenase, ETF dehydrogenase, dihydroorotate dehydrogenase, choline dehydrogenase, SQR, and proline dehydrogenase, which are present in lower amounts and are likely to be rate-limiting in an integrated electron transfer, are probably inserted in the respiratory chain by interaction through the CoQ pool [Genova and Lenaz, 2011]. A study addressed to this problem demonstrated that in mitochondria of brown fat the inhibition curve of glycerol phosphate-cytochrome $\mathrm{c}$ reductase is sigmoidal in the presence of myxothiazol and antimycin, suggesting the presence of a homogeneous CoQ pool between glycerol phosphate dehydrogenase (mtGPDH) and Complex III [Rauchová et al., 1997]. More recently, it has been shown that the delivery of electrons from mtGPDH to Complex III in mitochondria of human neutrophils takes place in the absence of supercomplex organization and of NADlinked respiration [van Raam et al., 2008]; this is in line with the notion that mtGPDH operates in mitochondria through the CoQ pool. Preliminary studies by BN-PAGE show that mtGPDH does not appear to be linked to any of the respiratory complexes [Genova ML, Rauchová M, Rauchová $\mathrm{H}$, unpubl. data] but associates in homooligomers as well as in supercomplexes that, however, do not contain either Complexes I, III, or IV [Mracek et al., 2014].

Since most authors agree that Complex II is mostly not physically interacting with Complex III in supercomplexes, also reverse electron transfer from succinate to $\mathrm{NAD}^{+}$, involving sequential interaction of Complexes II and I by means of CoQ, must take place by collisional interactions in the CoQ pool. The hyperbolic relation experimentally found by Gutman [1985] between the rate of reverse electron transfer and succinate oxidase is in complete accordance with the pool equation.

This observation poses a puzzling question [Lenaz and Genova, 2007]: if all or most Complex I units are associated with Complex III and the interaction of CoQ in the pool with the quinone-binding site in common between the 2 enzymes is necessarily slow, then how can $\mathrm{QH}_{2}$ reduced by Complex II interact from the pool with the CoQ site in Complex I at a rate compatible with the steady state kinetics of reverse electron transfer? The intriguing idea that Complex I may possess 2 different quinone-binding sites for direct and for reverse electron transfer, respec- 
tively, is compatible with the proposal that 2 different routes may exist for forward and reverse electron transfer within Complex I [Grivennikova et al., 2003]. These 2 sites might become alternatively accessible depending on the magnitude of the membrane potential. It must be noted that the ATP-driven reverse electron transfer from succinate to $\mathrm{NAD}^{+}$occurs in the presence of a high mitochondrial transmembrane proton motive force that, according to Piccoli et al. [2006], might be the physiological signal and at the same time the trigger causing the structural reorganization of the enzymatic complexes of the mitochondrial OXPHOS system. The model hypothesis depicted by Piccoli et al. [2006] from data on cytochrome oxidase was extended to other enzymes of the respiratory chain [Quarato et al., 2011], suggesting that also the I-III supercomplex would dissociate its constituting complexes under high $\Delta \mu_{\mathrm{H}}+$ condition, and this would no longer limit the access to the CoQ binding site in Complex I during reverse electron transfer.

The notion that probably all of Complex I is bound in a supercomplex would seem to exclude a function for the CoQ pool in electron transfer from NADH. This is not the case, as shown in the discussion in other sections of this review.

\section{Evidence for Separate Pools of CoQ}

As it was mentioned above, CoQ (ubiquinone) is required for the transfer of electrons from $\mathrm{NADH}$ - or $\mathrm{FAD}$ dependent enzymes to the respiratory Complex III within the inner mitochondrial membrane. Reduction/oxidation of CoQ is critical for energy production, redox balance, pyrimidine synthesis, amino acid and lipid metabolism, and indirectly for apoptosis control and calcium handling. For more than 40 years, it has generally been accepted that CoQ exerted its role by freely diffusing through the membrane and behaving as a homogenous pool available to any enzyme that required it, according to the RCM proposed to explain the organization of the mitochondrial electron transport chain [Lenaz and Genova, 2007]. The issue of CoQ compartmentalization is more than an academic controversy and has profound implications for our understanding of central physiological responses, as the adaptation of liver mitochondria to changing metabolic conditions, oxygen availability feed/ starvation periods, etc.

Some reports studying the reduction of $\mathrm{CoQ}$ in isolated mitochondria determined that succinate and $\mathrm{NADH}$ could reduce a limited and specific fraction of the total
CoQ pool [Jørgensen et al., 1985; Lass, 1998]. Moreover, an estimated $15-30 \%$ appeared to be bound to proteins (although the nature of the proteins was not determined), while the rest seemed to be free in the membrane [Lass and Sohal, 1999]. More recently, Rossignol and co-workers clearly demonstrated that the CoQ content in the mitochondrial inner membrane is not homogenous [Benard et al., 2008]. When they measured the levels of reduced CoQ and cytochrome $\mathrm{c}$ in state 3 isolated mitochondria, they found one CoQ fraction that was utilized during steady-state respiration, another fraction that acted as a reserve to maintain normal energy fluxes in case of perturbation (e.g. as a consequence of respiratory complex inhibition or mitochondrial disease), and a third fraction that is unable to participate in succinate-dependent respiration. This succinate non-utilizable pool was estimated to account for $79 \%$ of the total CoQ in muscle mitochondria and $21 \%$ in liver mitochondria. Although similar assays analyzing NADH dependent respiration have not been performed, these results are not compatible with a single CoQ pool.

Several reports have confirmed that respiratory supercomplexes contain also CoQ and that it is able to transfer electrons from Complex I to Complex III [Acín-Peréz et al., 2008; Althoff et al., 2011]. Recently, it was demonstrated that the physical assembly between Complexes I and III determines a preferential pathway for electrons mediated by a dedicated subset of CoQ molecules (we name them $\left.\mathrm{CoQ}_{\mathrm{NADH}}\right)$. This compartmentalization prevents significant cross talk between $\mathrm{NADH}$ oxidation (Complex I dependent) and FAD oxidation (CoQ $\mathrm{CAD}_{\mathrm{FAD}}$ pool) from any non-Complex I-dependent oxidation (if we assume that no further compartmentalization of the CoQ pool occurs), at least until the electrons reach cytochrome c. In that way, the CoQ pool behavior will fully apply to all enzymes that deliver electrons to the $\mathrm{CoQ}_{\mathrm{FAD}}$ (succinate dehydrogenase, glycerol-3-phosphate dehydrogenase, ETF-ubiquinone oxidoreductase, dihydroorotate dehydrogenase, choline dehydrogenase, SQR, and proline dehydrogenase). The segmentation of CoQ into the NADH- and the FAD-dedicated fractions has an unexpected consequence. Those Complex III molecules that physically interact with Complex I in the formation of supercomplexes are also dedicated to $\mathrm{CoQ}_{\mathrm{NADH}}$ oxidization $\left(\mathrm{CIII}_{\mathrm{NADH}}\right)$, while those Complex III molecules that are free of interaction with Complex I are mainly responsible for $\mathrm{CoQ}_{\mathrm{FAD}}$ oxidization. Complex I has a very high affinity for Complex III, to the extent that this association is preferred to the Complex I free state when the CI:CIII stoichiometry decreases abnormally due to the partial 
loss of Complex III. In this situation, $\mathrm{CoQ}_{\mathrm{NADH}}$ oxidization is preferentially maintained despite the risk of compromising the oxidation of $\mathrm{CoQ}_{\mathrm{FAD}}$ [Lapuente-Brun et al., 2013]. Therefore, the supercomplex organization of the mitochondrial electron transport chain has deeper functional consequences than expected. Adaptation to mitochondrial respiratory substrates that generate different proportions of NADH and FAD (as when mitochondria rely on fatty acids rather than glucose during fasting) requires adjustments to the capacity for electron transport via the NADH and FAD routes. Regulated modifications of the proportion of respiratory supercomplexes allow this adaptation [Lapuente-Brun et al., 2013], but how this change is produced and what would happen if this adaptation was impeded remain to be investigated. At present, no known molecular mechanism can be invoked to explain the exquisite regulation of the balance between NADH Complex III (Complex III dedicated to Complex I) and FAD Complex III. Further understanding of this behavior is of paramount importance.

\section{Biogenesis and Assembly of Supercomplexes}

A very relevant question derived from the plasticity model proposal is how the respiratory supercomplexes are generated and how this is related with the assembly of complexes themselves. This question was addressed by pulse-chase experiments, labeling the $13 \mathrm{mtDNA}$-encoded proteins and analyzing their time course incorporation into respiratory complexes and supercomplexes by BNGE/SDS-PAGE 2D electrophoresis [Acín-Peréz et al., 2008]. After $1 \mathrm{~h}$ of pulse- and short-chase periods for 0.5 and $2 \mathrm{~h}$, we could observe that most of the proteins were still in the process of being assembled in respiratory complexes and that they were progressing to higher-molecular-weight associations with time. This is the case for Complexes I, III, and IV. Thus, most of the signal corresponding to the mtDNA encoded Complex IV subunits (COI, COII, and COIII) had not reached the mature Complex IV until $12 \mathrm{~h}$ of chase. The same observation was true for the mtDNA-encoded subunits of Complex I and for the Complex III subunit cyt b. Complex V subunits behave differently, since all the radioactive signal for A6 and A8 was already incorporated in the mature Complex $\mathrm{V}$ even at very short chase periods. This is probably due to the fact that they incorporate at the end of the assembly process [Tzagoloff et al., 2004] and that this complex has a faster turnover. The labeling of large supercomplexes showed a different pattern depending on if they contained Complex IV or not. Thus, supercomplexes I/ III appear labeled as soon as after $0.5 \mathrm{~h}$ of chase. On the contrary, no labeling of supercomplexes I/III/IV was observed at 0.5 and $2 \mathrm{~h}$ of chase despite the fact that fully assembled Complex IV could be already observed. The labeling of the supercomplexes did not reach the characteristic pattern until $12 \mathrm{~h}$ of chase. These results support the idea that supercomplexes are structures formed by the specific and ordered association of individual complexes [Acín-Peréz et al., 2008]. A recent report proposed a different path for supercomplex formation [Moreno Lastres et al., 2012]. They used a different, although similar, experimental approach and depleted cells of OXPHOS complexes by treatment with doxycycline which reversibly inhibits mitochondrial translation. Then, upon drug removal, they followed complex and supercomplex assembly by BN-PAGE and immunodetection of individual proteins as markers of the overall complexes or subcomplexes. They also assumed that every co-migration in a gel is due to physical interaction between the co-migrating entities. This analysis led them to propose that the biogenesis of the respirasome involves the coordinated and sequential association of specific combinations of partially assembled respiratory complexes and free subunits. In other words, that Complex I is not fully assembled (it lacks at least the NADH dehydrogenase module) but is starting to recruit Complex III and Complex IV subunits until a final step where supercomplex III+IV and partially assembled Complex I is made. At this step the NADH dehydrogenase module of Complex I is added [Moreno Lastres et al., 2012]. This model, if right, has major implications because it concludes that Complex I cannot be fully assembled in the absence of Complex III and/ or IV. So, the respirasome assembly is intimately linked to the Complex I assembly. The model could be invoked to explain the lack of Complex I in the absence of Complex III [Acín-Peréz et al., 2004], IV [Diaz et al., 2005], or cytochrome b [Vempati et al., 2008]. However, it cannot explain the normal assembly, stability, and functionality of Complex I in cells lacking Complex IV [Balsa et al., 2012] and Complex III [Diaz et al., 2012a] in different culture conditions. It cannot explain either the stability or function of Complex I in fungi that lack Complex III and IV but naturally respire by AOX [Maas et al., 2009]. In addition, it cannot explain the existence of mice lacking respirasomes because they lost SCAFI [Lapuente-Brun et al., 2013].

On the contrary, the existence of protein factors that regulate the assembly of the different respiratory complexes into respirasomes is fully compatible with the se- 
quential formation of first complexes and then supercomplexes [Acín-Peréz et al., 2008]. Three independent reports recently described 2 related $S$. cerevisiae proteins (renamed by the 3 groups as RCF1 and RCF2) that might be important for the assembly between Complexes III and IV [Chen et al., 2011; Strogolova et al., 2012; Vukotic et al., 2012]. However, in contrast with SCAFI, ablation of RCF1 and RCF2 impairs overall respiration and growth quality in non-fermentable substrates. This could be interpreted as support for the solid model and therefore evidence against the fluid and the plasticity models. However, these proteins are required for assembly of the COX3 subunit into mature Complex IV, and when ablated, Complex IV assembly is impaired [Chen et al., 2011; Strogolova et al., 2012; Vukotic et al., 2012]. Two mammalian orthologs, HIG1A and HIG2A, have been identified for RCF1. HIG1A depletion has no influence on respiratory complexes or supercomplexes [Chen et al., 2011] and has been linked to the regulation of the mitochondrial $\gamma$-secretase [Hayashi et al., 2012]. HIG2A depletion induces a very moderate reduction in $\mathrm{CI}+\mathrm{III}+\mathrm{IV}$ supercomplexes but reduces Complex IV to the same extent as happens in yeast [Chen et al., 2011]. These proteins can therefore be understood as factors required for Complex IV function rather than specific supercomplex assembly factors. In contrast, SCAFI modulates the interaction between Complex III and IV without affecting their assembly or stability and is thus a true supercomplex assembly factor [Lapuente-Brun et al., 2013].

\section{Function of the Supercomplexes}

Four major roles have been attributed to the supercomplex organization of the mETC: (a) increased efficiency of electron flux, through substrate channeling or enhanced catalysis, (b) sequestration of reactive intermediates to prevent generation of ROS, (c) structural stabilization of individual respiratory complexes, and (d) structures in which Complex I is assembled and activated.

Next, we will discuss the experimental support obtained to confirm or discard the attributed roles.

\section{Kinetic Advantage: Channeling}

The primary proposed functional consequence of supercomplex assemblies in the respiratory chain was substrate channeling or enhanced catalysis in inter-complex electron transfer. Substrate channeling is the direct transfer of an intermediate between the active sites of 2 enzymes catalyzing consecutive reactions [Ovádi, 1991]; in the case of electron transfer this means direct transfer of electrons between 2 consecutive enzymes by successive reduction and re-oxidation of the intermediate without its diffusion in the bulk medium. In such a case, intercomplex electron transfer becomes indistinguishable from intra-complex electron transfer so that the so-called mobile intermediates, predicted to exhibit substrate-like behavior in the classic view of the RCM, would rather be buried in the interface between the 2 consecutive complexes.

Kinetic analysis allowed distinguishing the channeling from the random diffusional encounters: the problem for electron transfer in the respiratory chain was tackled for the first time by the pioneering work of Kröger and Klingenberg [1973]; subsequently flux control analysis was exploited by us with the precise aim of demonstrating channeling. In enzymatic supercomplexes, where the interactions between active sites are fixed and substrates and intermediates are channeled from one defined site to another without leaving the protein environment, the metabolic pathway would behave as a single enzyme unit, and inhibition of any of the enzyme components would elicit the same (total) flux control. In particular, in a system, in which the respiratory chain is totally dissociated from other components of the OXPHOS apparatus (i.e. ATP synthase, membrane potential, and carriers), such as open non-phosphorylating submitochondrial particles, the existence of a supercomplex would elicit a flux control coefficient near unity at any of the respiratory complexes, and the sum of all apparent coefficients would be $>1$ [Kholodenko and Westerhoff, 1993]. The flux control coefficients of the respiratory complexes (I, II, III, IV) were investigated using mitochondria of bovine heart and submitochondrial particles devoid of substrate permeability barriers and performing titrations with specific inhibitors of each complex [Bianchi et al., 2004]. Both Complexes I and III were found to be highly rate-controlling over $\mathrm{NADH}$ oxidation, a strong kinetic evidence suggesting the existence of functionally relevant association between the 2 complexes. On the contrary, Complex IV appears to be randomly distributed, although it is possible that if any stable interaction with Complex IV exists in mammalian mitochondria, it escaped detection most likely due to a pronounced abundance of molecules in a non-assembled form. Moreover, Complex II is fully rate-limiting for succinate oxidation, clearly indicating the absence of substrate channeling towards Complexes III and IV.

More recent studies by flux control analysis in intact and permeabilized cells also showed that the sum of apparent flux control coefficients of the 3 proton-motive 
respiratory complexes (I, III, and IV) exceeded 1, supporting the real existence of supercomplexes in vivo [Quarato et al., 2011; Kaambre et al., 2012]. In particular, Quarato et al. [2011] showed that the control coefficients were high under conditions of low membrane potential (state 3 or uncoupling) but much lower at membrane potential exceeding $180 \mathrm{mV}$ (state 4), suggesting that the supercomplexes may be in a dynamic equilibrium with the isolated complexes depending on the energetic state of the membrane, thus supporting a previous observation from the same laboratory [Piccoli et al., 2006]. The existence of a dynamic equilibrium of the respiratory complexes from an isolated form to supercomplex association supports the plasticity model [Acín-Peréz et al., 2008].

A direct demonstration of kinetic advantage in the CoQ region was demonstrated [Bianchi et al., 2003] by the striking rate increase of experimentally determined $\mathrm{NADH}$-cytochrome $\mathrm{c}$ reductase activity over the values calculated by the pool equation of Kröger and Klingenberg [1973] in Complex I-III proteoliposomes reconstituted at lipid:protein ratios $<10: 1$. Moreover when the same proteoliposomes at 1:1 lipid:protein ratios were treated with dodecyl-maltoside to destroy the supercomplex organization, the NADH-cytochrome c reductase activity fell dramatically, whereas both Complex I and Complex III individual activities were unchanged [Maranzana et al., 2013]; the same behavior was detected by treating bovine mitochondria with the detergent. Additional evidence for channeling between Complexes I and III derives from the demonstration that selective reduction of Complex III under a certain threshold induces a preferential deficiency in the transfer of electrons between Complexes II and III, while the transfer of electrons between Complex I and III remains unaltered [Lapuente-Brun et al., 2013].

While channeling in the I-CoQ- $\mathrm{III}_{2}$ supercomplex is ascertained, the role of cytochrome $\mathrm{c}$ in electron transfer between Complex III and IV is uncertain. Although the large amount of free Complex IV may have masked the electron channeling from III to IV in the supercomplex in the flux control experiments, the large majority of electron transfer must anyway escape direct transfer in the supercomplex. Since the flux control data established lack of control of Complex IV in electron transfer from NADH that is totally channeled to supercomplex-bound Complex III, this means that Complex III delivers electrons to Complex IV via a random cytochrome c pool and not via a fixed pathway within the I- $\mathrm{III}_{2}-\mathrm{IV}$ supercomplex. The lack of function of cytochrome $\mathrm{c}$ trapped in supercomplexes has been recently stressed by time-resolved analy-

CoQ Pool and Mitochondrial

Supercomplexes sis of oxidation of cytochrome $\mathrm{c}$ by its oxidase in intact yeast cells [Trouillard et al., 2011] that was proposed to be a random unrestricted process indicating the absence of functional microdomains. However, it is very difficult to extrapolate Trouillard et al's proposal to mammalian mitochondria for several reasons. First, the mETC of $S$. cerevisiae is very different from that of mammals because it lacks Complex I, and the analysis therefore relates only to assemblies containing Complexes III and IV. Second, the authors do not present a parallel analysis of the status of interaction between respiratory complexes. For their analysis, they poisoned the mETC with inhibitors of Complex III or with carbon monoxide (a Complex IV poison) and performed experiments in low oxygen, even exposing cells to the absence of oxygen. Unfortunately, they did not address the consequences of this rather strong manipulation on the structure and function of the mETC. Furthermore, they used several genetically modified strains for which they did not experimentally confirm the assumed structure of the mETC under their growth conditions. Moreover, these interpretations enter in frank contradiction with other studies in S. cerevisiae that propose that the mETC is a single functional unit [Boumans et al., 1998].

On the contrary, we demonstrated that the respiration capacity of mouse mitochondria is substantially modified by the organization of the respiratory complexes in dynamic superstructures [Lapuente-Brun et al., 2013]. In particular, we showed that when Complex IV is maintained permanently detached from supercomplexes by elimination of SCAFI, the maximum respiration activity of intact cells under glucose-rich medium parallels the maximum respiration activity obtained in the same cells by feeding directly electrons to Complex IV. On the contrary, if Complex IV is allowed to participate in supercomplexes, a significant proportion of Complex IV activity is no longer utilized by glucose driving respiration [Lapuente-Brun et al., 2013]. Moreover, we demonstrated in isolated liver mitochondria that the assembling of Complex IV in supercomplexes defines 3 types of Complex IV, one dedicated exclusively to receive electrons from NADH oxidation (forming supercomplex I+III+ IV), another dedicated to receive electrons from FADdependent enzymes (forming supercomplex III+IV), and a major third one that, being free Complex IV, is able to receive electrons from both $\mathrm{NADH}$ and FAD oxidization [Lapuente-Brun et al., 2013]. We also showed that an optimization in the simultaneous use of different carbon substrates (more physiological), the oxidization of which generates variable proportions of $\mathrm{NADH}$ versus $\mathrm{FADH}_{2}$, 
is achieved by modulation of the proportion of complexes and supercomplexes.

A different question concerns the physiological existence of electron transfer from Complex I to Complex III via the CoQ pool in addition or alternative to channeling in the supercomplex.

Complex I and Complex III, when mixed as concentrated solutions in detergent and then co-dialyzed, combine reversibly in a 1:1 molar ratio to form a Complex I-III unit (NADH-cytochrome $\mathrm{c}$ oxidoreductase) that contains equimolar FMN and cytochrome $c_{1}$ (clearly at difference with the supercomplexes found by BN-PAGE, where Complex III is present as a dimer) and 2-3 moles of ubiquinone-10 per mol of protein unit. In this unit, CoQ mobility is highly restricted suggesting that $\operatorname{CoQ}_{10}$ is effectively trapped between the component complexes in an environment that may be partly protein and partly derived from the lipid annuli of those complexes [Ragan and Heron, 1978]. However, CoQ-pool behavior could be restored, and Complex I and Complex III could be made to operate independently of each other by raising the concentrations of phospholipid and ubiquinone ( 2 -fold and 6-fold increase, respectively) in the concentrated mixture [Heron et al., 1978]. Heron et al., [1978] have proposed that, when phospholipid in excess of that needed to form an annulus is absent, relative mobility is lost, and complexes are frozen in their Complex I-III assembly, favoring a stable orientation of the site of reduction of ubiquinone with respect to the site of oxidation.

The analogous system described in the section 'Molecular Structure of Supercomplexes' and obtained by fusing a crude mitochondrial fraction enriched in Complex I and Complex III with different amounts of phospholipids and $\mathrm{CoQ}_{10}$, was used to discriminate whether the reconstituted protein fraction behaves as individual enzymes (CoQ-pool behavior) or as assembled supercomplexes depending on the experimental distances between the intramembranous particles [Lenaz et al., 1999]. The comparison of the experimentally determined NADHcytochrome $\mathrm{c}$ reductase activity with the values expected by theoretical calculation applying the pool equation showed overlapping results at phospholipid dilutions from 1:10 on, whereas at lower dilution $(<1: 5)$, pool behavior was not effective any more [Lenaz et al., 1999; Bianchi et al., 2003].

In addition, some studies in intact cells showed that an absence of supercomplexes, though diminishing electron transfer, allowed respiratory activity still to occur. Rosca et al. [2008] conducted a study on canine cardiac mitochondria in heart failure, induced experimentally by mi- cro-embolization. These mitochondria showed a severe decrease of the major $\mathrm{CI} / \mathrm{CIII}_{2} / \mathrm{CIV}$ supercomplex. Although the activity of individual Complexes I, III, and IV was normal, respiration with NAD-linked substrates in state 3 or after treatment with an uncoupler was strongly affected but still present. Neutrophil mitochondria may lack supercomplex organization; although they did not study mitochondrial integrated respiratory activities, van Raam et al. [2008] found that membrane potential induced by glutamate-malate was present, whereas lower than that induced by glycerol phosphate.

Thus, we can conclude from present knowledge that electron transfer between Complex I and Complex III can occur in the absence of supercomplex organization and it takes place by diffusional encounters of CoQ with the partner enzymes. This observation supports the plasticity model. In addition, it suggests that pathological conditions, in which the supercomplex organization is lost, are still compatible with electron transfer from $\mathrm{NADH}$, but this is made less efficient by resumption of the pool behavior [Lenaz and Genova, 2012].

The mechanism of electron transfer in supercomplexes is still uncertain. Ideally, we should have a detailed knowledge of the molecular structure of the interacting sites, but this knowledge is still lacking. Obviously we may have the extremes from close docking of the active sites with real inter-protein tunneling up to relatively long distances that may be covered either by important conformation changes or by restricted diffusion (microdiffusion) of the mobile components within the space between the 2 active sites; all of these alternatives have an obligate channeling between 2 fixed sites in common so that even the last situation, microdiffusion, would be quite distinguishable from pool behavior where the interaction of the mobile component may stochastically occur with a great number of possible sites located on several different protein targets which can be reached by random diffusion [Genova and Lenaz, 2011]. In the interaction between Complex I and Complex III within a supercomplex, if the sites are connected by CoQ microdiffusion, it is possible that it takes place within a lipid milieu, although we cannot exclude that the sites are put together by movement of CoQ on the protein or by movement of the protein itself. The previously described kinetic analysis cannot distinguish among different possible mechanisms of channeling.

In the $3 \mathrm{D}$ structure of the mitochondrial supercomplex $\mathrm{I}_{1} \mathrm{III}_{2} \mathrm{IV}_{1}$, which has recently been reported by Althoff et al. [2011], a unique arrangement of the 3 component complexes indicated the pathways along which ubiqui- 
none and cytochrome c can travel to shuttle electrons between their respective protein partners. In the above mentioned model, one of the Complex III monomers faces the lipid bilayer, while the other is surrounded by Complex I. Ubiquinol-binding sites are located between the $49-\mathrm{kDa}$ and the Complex I subunits near the first FeS cluster above the membrane in Complex I and the cytochrome b subunit in Complex III. Given that the shortest connection $(11.6 \mathrm{~nm})$ between those binding sites would run partly through the aqueous medium, a 13-nm trajectory through a gap within the supercomplex, which is filled with membrane lipid, is more likely (fig. 1). Althoff and colleagues also reported the presence of significant amounts of bound phospholipids in the purified supercomplex from mammalian mitochondria and demonstrated that cardiolipin is enriched in the supercomplex compared with total lipid of bovine heart. Moreover, HPLC analysis of the lipid extracts indicated that each supercomplex contains at least one molecule of ubiquinol [Althoff et al., 2011].

There are recent indications that supercomplex association may provide further kinetic advantages besides substrate channeling. The study of Schäfer et al. [2007] showed that a supercomplex comprising cytochrome oxidase $\left(\mathrm{I}_{1} \mathrm{III}_{2} \mathrm{IV}_{1}\right)$ had higher Complex I and Complex III activities than the supercomplex devoid of the terminal oxidase $\left(\mathrm{I}_{1} \mathrm{III}_{2}\right)$; evidently, the presence of Complex IV modifies the conformation of the partner complexes in such a way to enhance their catalytic activity. Likewise, Hildebrandt [2011] showed that supercomplex dissociation abolishes the protective effect of dehydroascorbic acid on sulphide toxicity to cytochrome oxidase, suggesting a conformational effect of supramolecular association on the allosteric properties of cytochrome oxidase.

\section{Supercomplexes and ROS Generation}

Indirect circumstantial evidence suggests that supercomplex assembly may limit the extent of superoxide generation by the respiratory chain. Panov et al. [2007] proposed that the respirasome helps to maintain the redox components of the complexes in the oxidized state through the facilitation of electron flow by channeling, thus limiting ROS formation. Similarly, Seelert et al. [2009] also suggested that facilitation of electron transfer by channeling may limit the detrimental generation of ROS.

Two potential sites for oxygen reduction exist in Complex I, represented by FMN and iron-sulphur cluster N2; controversial results from different laboratories working either on isolated Complex I or on mitochondrial mem-

CoQ Pool and Mitochondrial

Supercomplexes branes generally indicated that $\mathrm{N} 2$ as a source of ROS would be predominant in membrane particles, whereas FMN might become available after Complex I isolation [Lenaz and Genova, 2010]. A reasonable hypothesis is that FMN becomes exposed to oxygen only when Complex I is dissociated from Complex III. Although the molecular structure of the individual complexes does not allow to envisage a close apposition of the matrix arm of Complex I, where FMN is localized with either Complex III or IV, the actual shape of the $\mathrm{I}_{1} \mathrm{III}_{2} \mathrm{IV}_{1}$ supercomplex from bovine heart suggests a slightly different conformation of Complex I in the supercomplex [Schäfer et al., 2007], showing a smaller angle of the matrix arm with the membrane arm and a higher bending towards the membrane (and presumably Complex III) in line with the notion that Complex I may undergo important conformational changes [Radermacher et al., 2006]. Moreover, the observed destabilization of Complex I in the absence of a supercomplex may render the $51-\mathrm{kDa}$ subunit containing the FMN more 'loose', allowing it to interact with oxygen.

This issue was directly addressed by testing the ROS generation by Complex I in 2 experimental systems in which the supramolecular organization of the respiratory assemblies was impaired by (a) treatment either of mitochondria of bovine heart or liposome-reconstituted supercomplex I-III with dodecyl-maltoside, and (b) reconstitution of Complexes I and III at a high phospholipids to protein ratio. The results of our investigation provided experimental evidence that the production of ROS is strongly increased in either model, supporting the view that disruption or prevention of the association between Complex I and Complex III by different means enhances the generation of superoxide from Complex I [Maranzana et al., 2013].

The large evidence that supercomplexes physiologically exist in dynamic equilibrium with isolated complexes raises the puzzling question that also ROS generation may be subjected to physiological changes; the suggestion that at high mitochondrial membrane potential supercomplexes are dissociated [Quarato et al., 2011] is compatible with the increase of ROS generation under the same conditions [Skulachev, 1996]. It is tempting to suggest that these changes may be aimed to regulate ROS levels in the cell in view of the well-documented role of ROS in cellular redox signaling [Murphy, 2012].

The elevated production of ROS in mouse lung fibroblasts lacking the Rieske iron-sulfur protein of Complex III and hence being devoid of the supercomplexes is in agreement with this view [Diaz et al., 2012b]. Other ex- 
amples of enhanced ROS generation by dissociation of supramolecular units exist in the literature: for succinate cytochrome $\mathrm{c}$ reductase [Zhang et al., 1998] and for ETF linked to medium-chain acylCoA dehydrogenase [Rodrigues and Gomes, 2012].

The relevance of the above observations to physiopathological events is supported by several studies in cellular models and in animals. Thus, in mouse fibroblasts expressing the activated form of the K-ras oncogene, a strong decrease of high molecular weight supercomplexes correlates with higher ROS generation [Baracca et al., 2010; Lenaz et al., 2010]. In heart failure, an increased production of ROS, either from mitochondria or from NADPH oxidase, drives signaling pathways that mediate the cardiac remodeling [Ide et al., 2000; Anilkumar et al., 2009; Chen and Keaney, 2012]. In an experimental model of heart failure, the decrease of oxidative phosphorylation has been associated with a decrease of respiratory supercomplexes [Rosca et al., 2008, 2011; Rosca and Hoppel, 2009]. Lymphoblasts from patients affected by Barth syndrome due to genetic loss of Tafazzin, an enzyme involved in cardiolipin remodeling, have altered mitochondrial supercomplexes [McKenzie et al., 2006]; likewise, in an yeast experimental model of mutated Tafazzin, an increased oxidative stress in response to ethanol was observed [Chen et al., 2008].

Aging is accompanied by a decline of supercomplex association [Gómez et al., 2009; Frenzel et al., 2010] and is generally associated with increased ROS and oxidative damage [for a recent review, see Cui et al., 2012]. Ageassociated destabilization of respiratory supercomplexes may therefore be important for the development of the mitochondrial aging phenotype by means of impaired bioenergetics and enhanced ROS production [Gómez and Hagen, 2012].

\section{Stability and Assembly}

Analysis of the state of supercomplexes in human patients with an isolated deficiency of Complex III [AcínPeréz et al., 2004; Schägger et al., 2004] and in cultured cell models harboring Complex III [Acín-Peréz et al., 2009b], Complex IV [Diaz et al., 2006], or cytochrome c [Vempati et al., 2008] depletion led to the proposal that the formation of respirasomes may be essential for the assembly/stability of Complex I. Genetic alterations causing a loss of Complex III induced a secondary loss of Complex I, therefore primary Complex III assembly deficiencies presented as Complex III/I defects [Lamantea et al., 2002; Bruno et al., 2003; Acín-Peréz et al., 2009b]. Conversely, Complex III stability was not influ- enced by the absence of Complex I [Acín-Peréz et al., 2009b].

Under this assumption, D'Aurelio et al. [2006] studied the complementation of mtDNA in human cells by fusing 2 cell lines, one containing a homoplasmic mutation in a subunit of the respiratory chain Complex IV, COX-I, and the other with a distinct homoplasmic mutation in a subunit of Complex III, cytochrome b. Upon cell fusion, respiration was recovered in correlation with the presence of supercomplexes containing Complexes I, III, and IV. From these findings, supercomplex assembly was proposed as a necessary step for respiration. Together with that, deletion of the Ndufs 4 gene, encoding an accessory subunit of Complex I, in mouse tissues results in decreased stability and activity of Complex I; however, the portion of Complex I that is still active is bound in the supercomplexes, indicating that the supramolecular association improves the stability of Complex I [Calvaruso et al., 2011].

Animal models of the effects of Complex III and Complex IV mutations on Complex I should prove useful for a better understanding of the role of supercomplexes. Two studies in Caenorhabditis elegans have recently been published on this aspect. In the first, a knockdown of the predicted homologues of COX-IV and COX-Va in the nematode showed that intrinsic Complex I enzymatic activity is dependent on the presence of Complex IV, despite no overall decrease in the amount of Complex I [Suthammarak et al., 2009]. In a further study in C. elegans, the same group showed that Complex III defects inhibit Complex I by several different mechanisms involving supercomplex destabilization [Suthammarak et al., 2010]. Mutant analysis revealed that Complex III affects supercomplex I-III-IV formation by acting as an assembly/stabilizing factor. Also, an mtDNA mutation affecting Complex III, $c t b-1$, inhibits Complex I function by weakening the interaction of Complex IV in supercomplex I-III-IV. Other Complex III mutations inhibit Complex I function either by decreasing the amount of Complex I (isp-1) or its assembly as the most active supramolecular form, the I-III-IV supercomplex (isp-1;ctb-1). It is suggested that allosteric interactions involve all 3 complexes within the supercomplex and are necessary for maximal enzymatic activities.

A recent study on fibroblasts lacking the Rieske iron sulphur protein questioned that the physical association of Complex I into supercomplexes per se would explain all the above results. Thus, it pointed out a different mechanism for Complex I destabilization in the absence of the Complexes III or IV, i.e. in an enhanced ROS gen- 
eration. Complex I would be particularly sensitive to ROS damage. Therefore, further research is needed to clarify this point.

\section{CoQ Saturation Kinetics: Is There a Contradiction?}

As previously described, Complex I is almost totally associated in a supercomplex with Complex III, with electron channeling of bound CoQ in the boundary between the 2 complexes. The CoQ pool is, however, directly required for electron transfer from Complex II to Complex III.

On the other hand, the finding that Complex I is almost totally associated in a supercomplex with Complex III seems to exclude a role for the CoQ pool in physiological electron transfer between these 2 complexes. Surprisingly, strong evidence exists that $\mathrm{NADH}$-cytochrome $\mathrm{c}$ reductase activity follows saturation kinetics with respect to CoQ.

The relation between electron transfer rate and CoQ concentration was seen for NADH and succinate oxidation in reconstituted systems [Estornell et al., 1992] and in phospholipid-enriched mitochondria [Schneider et al., 1982]. Direct titrations of CoQ-depleted mitochondria reconstituted with different CoQ supplements yielded a $\mathrm{K}_{\mathrm{m}}$ of NADH oxidation for $\mathrm{Q}_{\mathrm{t}}$ in the range of $2-5 \mathrm{nmol} /$ $\mathrm{mg}$ mitochondrial protein [Estornell et al., 1992], corresponding to a $\mathrm{Q}_{t}$ concentration of $4-10 \mathrm{mM}$ in the lipid bilayer. The $\mathrm{K}_{\mathrm{m}}$ for $\mathrm{CoQ}_{10}$ of NADH-cytochrome $\mathrm{c}$ reductase was found to be much higher than that of succinate-cytochrome $c$ reductase. A direct study on a reconstituted mitochondrial fraction containing Complexes I and III showed that the experimental rate of NADH-cytochrome $\mathrm{c}$ reductase was hyperbolically related to the content of $\mathrm{CoQ}_{10}$, with an apparent $\mathrm{K}_{\mathrm{m}}$ in the same range as in mitochondria [Lenaz et al., 1999].

Analysis of the literature shows that the physiological CoQ content of several types of mitochondria is in the range of the $\mathrm{K}_{\mathrm{m}}$ for NADH oxidation and, therefore, not saturating for this activity [Battino et al., 1990]. However, this does not exclude that free CoQ in the pool is also necessary for proper channeling between the complexes. In fact, the bound inter-complex quinone that allows electron flow directly from Complex I to Complex III must be in a dissociation equilibrium with the CoQ pool, so that its amount, at steady state, would be dictated by the size of the pool: this equilibrium explains the saturation kinetics for total ubiquinone exhibited by the integrated activity of Complex I and Complex III and the decrease of respira-

CoQ Pool and Mitochondrial

Supercomplexes tory activities in mitochondria fused with phospholipids with subsequent dilution of the CoQ pool. To be in agreement with the experimental observation obtained by metabolic flux analysis, this proposition requires that the dissociation rate constants $\left(\mathrm{k}_{\text {off }}\right)$ of bound CoQ be considerably slower than the rates of inter-complex electron transfer via the same bound quinone molecules [Lenaz and Genova, 2010; Genova and Lenaz, 2011]. The high apparent $\mathrm{K}_{\mathrm{m}}$ for $\mathrm{CoQ}_{10}$ in NADH oxidation is in line with this postulation. The observation by Schneider et al. [1982] that dilution of the inner membrane proteins with phospholipids lowers electron transfer and the effect is reversed by CoQ addition is in line with this concept. Earlier studies by Heron et al. [1978] also reported that endogenous ubiquinone-10 leaks out of the Complex I-III unit when extra phospholipid is present, causing a decrease in activity that could be alleviated by adding more ubiquinone. It is likely that the function of the large amount of ubiquinone in the natural membrane may be, therefore, to maintain the ubiquinone-10 content in the supercomplex unit when it is formed.

\section{What We Expect from CoQ Deficiency and Supplementation}

Coenzyme Q10 supplementation is able to restore mitochondrial respiration in primary $\mathrm{CoQ}_{10}$ deficiencies and is beneficial in several diseases in which a secondary CoQ deficiency is usually postulated; in this latter case, a bioenergetic deficit is not always apparent, and the health improvements are ascribed also to other properties of the quinone, such as its antioxidant activity.

Is the existence and role of supercomplexes compatible with the interpretation of the beneficial effects of exogenous $\mathrm{CoQ}_{10}$ administration on bioenergetic grounds? There is evidence, mainly indirect, that exogenous orally administered $\mathrm{CoQ}_{10}$ may be incorporated into mitochondria, at least in conditions of partial CoQ tissue deficiency, where it may enhance electron transfer and ATP synthesis with improvement of the disease both in human and animal studies, e.g. in genetic $\mathrm{CoQ}_{10}$ deficiency [Rötig et al., 2000; Di Giovanni et al., 2001; Quinzii and Hirano, 2010], cardiac failure [Rosenfeldt et al., 2005; Molyneux et al., 2009], Parkinson's disease [Matthews et al., 1998], Alzheimer's disease [Yang et al., 2010], Friedreich's ataxia [Hart et al., 2005], and aging [Xu et al., 2010]. The major problem of $\mathrm{CoQ}_{10}$ administration is its low bioavailability due to its extreme hydrophobicity [Beg et al., 2010]. A water-soluble formulation (Qter) has recently 


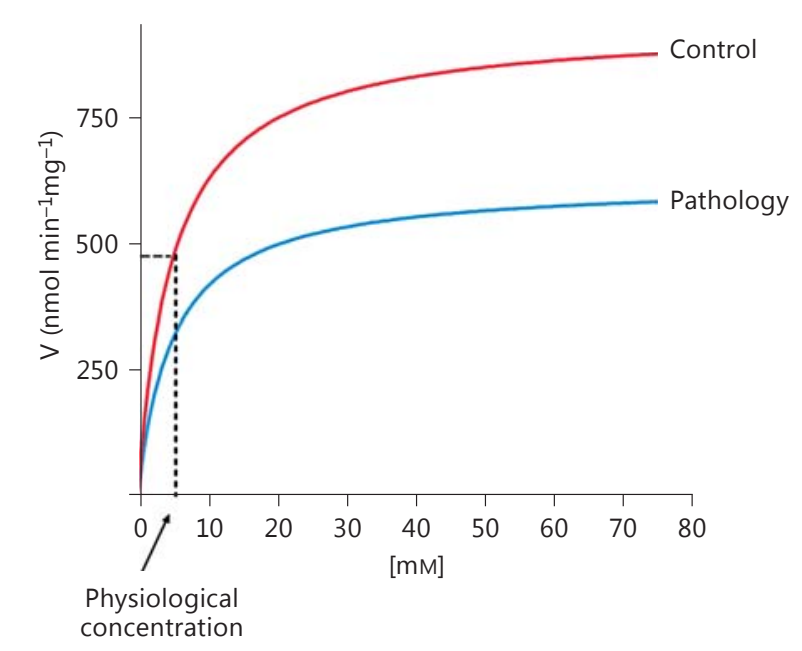

Fig. 3. Coenzyme Q supplementation restores NADH oxidation impaired by causes different from CoQ deficiency. The figure shows a hyperbolic curve of $\mathrm{NADH}$ oxidation rate (expressed as $\mathrm{nmol} /(\mathrm{min} \times \mathrm{mg})$ protein) as a function of CoQ10 concentration (expressed in $\mathrm{mM}$ in the membrane lipids). The $\mathrm{K}_{\mathrm{m}}$ of $\mathrm{NADH}$ oxidation for CoQ10 was found to be in the range 5-10 mM in the lipid phase [Estornell et al., 1992] which is about the physiological concentration in most types of mitochondria. Being in the firstorder arm of the curve, an increase in CoQ concentration is bound to enhance NADH oxidation both in normal mitochondria (red) and in mitochondria with decreased respiration (blue).

been shown to be easily incorporated into cultured cells and their mitochondria, enhancing respiration and antioxidant properties [Bergamini et al., 2012]. The same formulation was found to improve grip strength and to inhibit apoptosis in aged rats [Xu et al., 2010].

The existence of I-III supercomplexes where only inter-complex bound CoQ is active by channeling electrons from Complex I to Complex III is apparently incompatible with a dose-dependent effect of added $\mathrm{CoQ}_{10}$; however, the notion that inter-complex bound CoQ is in a chemical equilibrium with CoQ in the pool is sufficient to explain the improved cell bioenergetics upon addition of exogenous CoQ. It is expected that even a slight decrease of CoQ content in the membrane is sufficient to dissociate part of the quinone from the supercomplex thus decreasing the rate of electron channeling.

Kinetic studies in CoQ-depleted mitochondria [Estornell et al., 1992] showed that $\mathrm{CoQ}_{10}$ concentration in the inner membrane is in the range of its apparent $\mathrm{K}_{\mathrm{m}}$ in the $\mathrm{NADH}$ oxidation. This means that $\mathrm{CoQ}$ is physiologically not saturating and that increasing $\mathrm{CoQ}$ concentration in the membrane is bound to enhance respiratory activity: this is indeed what was found in the study with the watersoluble formulation quoted above [Bergamini et al., 2012]. Addition of Qter to cultured cells increased CoQ concentration in their mitochondria and significantly enhanced respiratory rates.

The fact of $\mathrm{CoQ}_{10}$ not being saturating in NADH oxidation should also drive a further consequence of improving respiration even when the defect is due to reasons different from CoQ deficiency, e.g. a defect of Complex I. This is theoretically shown in figure 3. A direct study by Estornell et al. [1997] demonstrated that a Complex I failure in vitamin A-deficient rats could be counteracted by an increase of $\mathrm{CoQ}_{10}$. This is the most likely explanation why $\mathrm{CoQ}$ was found to ameliorate several disease states even if a CoQ deficiency was not demonstrated.

What are the mechanisms of the beneficial effects of administered CoQ? The bioenergetic improvement due to enhanced electron transfer is certainly the major mechanism in primary CoQ deficiencies; however, it may be considered in all cases where a secondary deficiency takes place, as in heart failure and aging. An antioxidant effect may be at the basis of amelioration of symptoms in several pathological states. However, the direct antiapoptotic action exerted by its effect on the permeability transition pore should be considered in the case of degenerative diseases.

\section{Acknowledgements}

Work from the laboratory of G.L. was supported by grants from MUR, Rome Italy. Research of J.A.E. was supported by grants from the Ministerio de Ciencia e Innovación (SAF2012-32776 \& CSD2007-00020), the European Union (Marie Curie MEET-ITN, GA ñ: 317433), Comunidad Autónoma de Madrid (S2010/BMD2402), The CNIC is supported by the Ministerio de Economía y Competitividad and the Pro-CNIC Foundation.

\footnotetext{
References $\longrightarrow$ Acín-Peréz R, Bayona-Bafaluy MP, FernándezSilva P, Moreno-Loshuertos R, Pérez-Martos A, et al: Respiratory complex III is required to maintain complex I in mammalian mitochondria. Mol Cell 13:805-815 (2004).

-Acín-Peréz R, Fernández-Silva P, Peleato ML, Pérez-Martos A, Enriquez JA: Respiratory active mitochondrial supercomplexes. Mol Cell 32:529-539 (2008).

-Acín-Peréz R, Salazar E, Kamenetsky M, Buck J Levin LR, Manfredi G: Cyclic AMP produced inside mitochondria regulates oxidative phosphorylation. Cell Metab 9:265-276 (2009a).
}

Enriquez/Lenaz 
Acín-Peréz R, Salazar E, Brosel S, Yang H, Schon EA, Manfredi G: Modulation of mitochondrial protein phosphorylation by soluble adenylyl cyclase ameliorates cytochrome oxidase defects. EMBO Mol Med 1:392-406 (2009b).

Acín-Peréz R, Gatti DL, Bai Y, Manfredi G: Protein phosphorylation and prevention of cytochrome oxidase inhibition by ATP: coupled mechanisms of energy metabolism regulation. Cell Metab 13:712-719 (2011a).

Acín-Peréz R, Russwurm M, Gunnewig K, Gertz $\mathrm{M}$, Zoidl G, et al: A phosphodiesterase 2A isoform localized to mitochondria regulates respiration. J Biol Chem 286:30423-30432 (2011b).

-Althoff T, Mills DJ, Popot JL, Kühlbrandt W: Arrangement of electron transport chain components in bovine mitochondrial supercomplex $\mathrm{I}_{1} \mathrm{III}_{2} \mathrm{IV}_{1}$. EMBO J 30:4652-4664 (2011).

-Anilkumar N, Sirker A, Shah AM: Redox sensitive signaling pathways in cardiac remodeling, hypertrophy and failure. Front Biosci 14:31683187 (2009).

- Balsa E, Marco R, Perales-Clemente E, Szklarczyk $\mathrm{R}$, Calvo E, et al: NDUFA4 is a subunit of complex IV of the mammalian electron transport chain. Cell Metab 16:378-386 (2012).

- Baracca A, Chiaradonna F, Sgarbi G, Solaini G, Alberghina L, Lenaz G: Mitochondrial complex I decrease is responsible for bioenergetic dysfunction in K-ras transformed cells. Biochim Biophys Acta 1797:314-323 (2010).

- Battino M, Ferri E, Gorini A, Villa RF, Rodriguez Huertas JF, et al: Natural distribution and occurrence of coenzyme $\mathrm{Q}$ homologues. Membr Biochem 9:179-190 (1990).

Beg S, Javed S, Kohli K: Bioavailability enhancement of coenzyme Q10: an extensive review of patents. Recent Pat Drug Deliv Formul 4: 245-255 (2010).

-Bellomo F, Piccoli C, Cocco T, Scacco S, Papa F, et al: Regulation by the cAMP cascade of oxygen free radical balance in mammalian cells. Antioxid Redox Signal 8:495-502 (2006).

- Benard G, Faustin B, Galinier A, Rocher C, Bellance N, et al: Functional dynamic compartmentalization of respiratory chain intermediate substrates: implications for the control of energy production and mitochondrial diseases. Int J Biochem Cell Biol 40:1543-1554 (2008).

- Bergamini C, Moruzzi N, Sblendido A, Lenaz G, Fato R: A water soluble CoQ10 formulation improves intracellular distribution and promotes mitochondrial respiration in cultured cells. PLoS ONE 7:e33712 (2012).

- Bianchi C, Fato R, Genova ML, Parenti Castelli G, Lenaz G: Structural and functional organization of Complex I in the mitochondrial respiratory chain. Biofactors 18:3-9 (2003).

- Bianchi C, Genova ML, Parenti Castelli G, Lenaz G: The mitochondrial respiratory chain is partially organized in a supercomplex assembly: kinetic evidence using flux control analysis. J Biol Chem 279:36562-36569 (2004).
Boumans H, Grivell LA, Berden JA: The respiratory chain in yeast behaves as a single functional unit. J Biol Chem 273:4872-4877 (1998).

- Böttinger L, Horvath SE, Kleinschroth T, Hunte C, Daum G, et al: Phosphatidylethanolamine and cardiolipin differentially affect the stability of mitochondrial respiratory chain supercomplexes. J Mol Biol 423:677-686 (2012).

Brandner K, Mick DU, Frazier AE, Taylor RD, Meisinger C, Rehling P: Taz1, an outer mitochondrial membrane protein, affects stability and assembly of inner membrane protein complexes: implications for Barth syndrome. Mol Biol Cell 16:5202-5214 (2005).

-Bruno C, Santorelli FM, Assereto S, Tonoli E, Tessa A, et al: Progressive exercise intolerance associated with a new muscle-restricted nonsense mutation (G142X) in the mitochondrial cytochrome $b$ gene. Muscle Nerve 28:508-511 (2003).

Calvaruso MA, Willems P, van den Brand M, Valsecchi F, Kruse S, et al: Mitochondrial complex III stabilizes complex I in the absence of NDUFS4 to provide partial activity. Hum Mol Genet 21:115-120 (2011).

Castellani M, Covian R, Kleinschroth T, Anderka O, Ludwig B, et al: Direct demonstration of half-of-the-sites reactivity in the dimeric cytochrome bcl complex: enzyme with one inactive monomer is fully active but unable to activate the second ubiquinol oxidation site in response to ligand binding at the ubiquinone reduction site. J Biol Chem 285:502-510 (2010).

-Chen K, Keaney JF: Evolving concepts of oxidative stress and reactive oxygen species in cardiovascular disease. Curr Atheroscler Rep 14: 476-483 (2012).

-Chen S, He Q, Greenberg ML: Loss of tafazzin in yeast leads to increased oxidative stress during respiratory growth. Mol Microbiol 68: 1061-1072 (2008).

-Chen YC, Taylor EB, Dephoure N, Heo JM, Tonhato $\mathrm{A}$, et al: Identification of a protein mediating respiratory supercomplex stability. Cell Metab 15:348-360 (2011)

Claypool SM, Koehler CM: The complexity of cardiolipin in health and disease. Trends Biochem Sci 37:32-41 (2012).

Claypool SM, Oktay Y, Boontheung P, Loo JA, Koehler CM: Cardiolipin defines the interactome of the major ADP/ATP carrier protein of the mitochondrial inner membrane. J Cell Biol 182:937-950 (2008).

-Cui H, Kong Y, Zhang H: Oxidative stress, mitochondrial dysfunction, and aging. J Signal Transduct 2012:646354 (2012).

Dalmonte ME, Forte E, Genova ML, Giuffrè A, Sarti P, Lenaz G: Control of respiration by cytochrome c oxidase in intact cells: role of the membrane potential. J Biol Chem 284:3233132335 (2009).

D’Aurelio M, Gajewski CD, Lenaz G, Manfredi G: Respiratory chain supercomplexes set the threshold for respiration defects in human mtDNA mutant cybrids. Hum Mol Genet 15: 2157-2169 (2006).
Deng N, Zhang J, Zong C, Wang Y, Lu H, et al: Phosphoproteome analysis reveals regulatory sites in major pathways of cardiac mitochondria. Mol Cell Proteomics 10:M110.000117 (2011)

de Rasmo D, Panelli D, Sardanelli AM, Papa S: cAMP-dependent protein kinase regulates the mitochondrial import of the nuclear encoded NDUFS4 subunit of complex I. Cell Signal 20:989-997 (2008)

Diaz F, Thomas CK, Garcia S, Hernandez D, Moraes CT: Mice lacking COX10 in skeletal muscle recapitulate the phenotype of progressive mitochondrial myopathies associated with cytochrome $\mathrm{c}$ oxidase deficiency. Hum Mol Genet 14:2737-2748 (2005).

- Diaz F, Fukui H, Garcia S, Moraes CT: Cytochrome coxidase is required for the assembly/ stability of respiratory complex I in mouse fibroblasts. Mol Cell Biol 26:4872-4881 (2006).

Diaz F, Garcia S, Padgett KR, Moraes CT: A defect in the mitochondrial complex III, but not complex IV, triggers early ROS-dependent damage in defined brain regions. Hum Mol Genet 21:5066-5077 (2012a).

Diaz F, Enriquez JA, Moraes CT: Cells lacking Rieske iron-sulfur protein have a reactive oxygen species-associated decrease in respiratory complexes I and IV. Mol Cell Biol 32: 415-429 (2012b).

Dieteren CE, Willems PH, Vogel RO, Swarts HG, Fransen J, et al: Subunits of mitochondrial complex I exist as part of matrix- and membrane-associated subcomplexes in living cells. J Biol Chem 283:34753-34761 (2008).

- Di Giovanni S, Mirabella M, Spinazzola A, Crociani P, Silvestri G, et al: Coenzyme Q10 reverses pathological phenotype and reduces apoptosis in familial CoQ10 deficiency. Neurology 57:515-518 (2001)

-Dudkina NV, Eubel H, Keegstra W, Boekema EJ, Braun HP: Structure of a mitochondrial supercomplex formed by respiratory-chain complexes I and III. Proc Natl Acad Sci USA 102:3225-3229 (2005).

-Dudkina NV, Kudryashev M, Stahlberg H, Boekema EJ: Interaction of complexes I, III, and IV within the bovine respirasome by single particle cryoelectron tomography. Proc Natl Acad Sci USA 108:15196-15200 (2011).

- Estornell E, Fato R, Castelluccio C, Cavazzoni M, Parenti Castelli G, Lenaz G: Saturation kinetics of coenzyme Q in NADH and succinate oxidation in beef heart mitochondria. FEBS Lett 311:107-109 (1992).

Estornell E, Tormo JR, Barber T: A deficiency in respiratory complex I in heart mitochondria from vitamin A-deficient rats is counteracted by an increase in coenzyme Q. Biochem Biophys Res Commun 233:451-454 (1997).

Frenzel M, Rommelspacher H, Sugawa MD, Dencher NA: Ageing alters the supramolecular architecture of OxPhos complexes in rat brain cortex. Exp Gerontol 45:563-572 (2010) 
-Fry M, Green DE: Energized cation transport by complex III (ubiquinone-cytochrome C reductase). Biochem Biophys Res Commun 97: 852-859 (1980).

Fry M, Green DE: Cardiolipin requirement for electron transfer in complex I and III of the mitochondrial respiratory chain. J Biol Chem 256:1874-1880 (1981).

-Genova ML, Lenaz G: New developments on the functions of coenzyme Q in mitochondria. Biofactors 37:330-354 (2011).

-Genova ML, Baracca A, Biondi A, Casalena G, Faccioli M: Is supercomplex organization of the respiratory chain required for optimal electron transfer activity? Biochim Biophys Acta 1777:740-746 (2008).

-Gong J, Hoyos B, Acín-Peréz R, Vinogradov V, Shabrova E, et al: Two protein kinase $\mathrm{C}$ isoforms, $\delta$ and $\varepsilon$, regulate energy homeostasis in mitochondria by transmitting opposing signals to the pyruvate dehydrogenase complex. FASEB J 26:3537-3549 (2012).

Gómez LA, Hagen TM: Age-related decline in mitochondrial bioenergetics: does supercomplex destabilization determine lower oxidative capacity and higher superoxide production? Semin Cell Dev Biol 23:758-767 (2012).

-Gómez LA, Monette JS, Chavez JD, Maier CS, Hagen TM: Supercomplexes of the mitochondrial electron transport chain decline in the aging rat heart. Arch Biochem Biophys 490:3035 (2009).

Green DE, Tzagoloff A: The mitochondrial electron transfer chain. Arch Biochem Biophys 116:293-304 (1966).

- Grimsrud PA, Carson JJ, Hebert AS, Hubler SL, Niemi NM, et al: A quantitative map of the liver mitochondrial phosphoproteome reveals posttranslational control of ketogenesis. Cell Metab 16:672-683 (2012).

- Grivennikova VG, Roth R, Zakharova NV, Hägerhäll C, Vinogradov AD: The mitochondrial and prokaryotic proton-translocating $\mathrm{NADH}$ :ubiquinone oxidoreductases: similarities and dissimilarities of the quinone-junction sites. Biochim Biophys Acta 1607:79-90 (2003).

Gutman M: Kinetic analysis of electron flux through the quinones in the mitochondrial system, in Lenaz G, Wiley C (eds): Coenzyme Q: Biochemistry, Bioenergetics, and Clinical Applications of Ubiquinone, pp 215-234 (UK: John Wiley \& Sons Inc, Hoboken 1985).

Hackenbrock CR, Chazotte B, Gupte SS: The random collision model and a critical assessment of diffusion and collision in mitochondrial electron transport. J Bioenerg Biomembr 18: 331-368 (1986).

- Hart PE, Lodi R, Rajagopalan B, Bradley JL, Crilley JG, et al: Antioxidant treatment of patients with Friedreich ataxia: four-year follow-up. Arch Neurol 62:621-626 (2005).

-Hatefi Y, Haavick AG, Fowler LR, Griffiths DE: Studies on the electron transfer system. XLII. Reconstitution of the electron transfer system. J Biol Chem 237:2661-2669 (1962a).
Hatefi Y, Haavick AG, Griffiths DE: Studies on the electron transfer system. XL. Preparation and properties of mitochondrial DPNH-coenzyme Q reductase. J Biol Chem 237:16761680 (1962b).

Hayashi $\mathrm{H}$, Nakagami H, Takeichi M, Shimamura M, Koibuchi N, et al: HIG1, a novel regulator of mitochondrial $\gamma$-secretase, maintains normal mitochondrial function. FASEB J 26: 2306-2317 (2012)

-Helling S, Vogt S, Rhiel A, Ramzan R, Wen L, et al: Phosphorylation and kinetics of mammalian cytochrome $\mathrm{c}$ oxidase. Mol Cell Proteomics 7:1714-1724 (2008).

Heron C, Ragan CI, Trumpower BL: The interaction between mitochondrial NADH-ubiquinone oxidoreductase and ubiquinol-cytochrome c oxidoreductase. Restoration of ubiquinone-pool behaviour. Biochem J 174: 791-800 (1978).

- Hébert Chatelain E: Src kinases are important regulators of mitochondrial functions. Int J Biochem Cell Biol 45:90-98 (2013).

Hildebrandt TM: Modulation of sulfide oxidation and toxicity in rat mitochondria by dehydroascorbic acid. Biochim Biophys Acta 1807: 1206-1213 (2011)

Ide T, Tsutsui H, Kinugawa S, Suematsu N, Hayashidani S, et al: Direct evidence for increased hydroxyl radicals originating from superoxide in the failing myocardium. Circ Res 86:152-157 (2000).

Iuso A, Scacco S, Piccoli C, Bellomo F, Petruzzella V, et al: Dysfunctions of cellular oxidative metabolism in patients with mutations in the NDUFS1 and NDUFS4 genes of complex I. J Biol Chem 281:10374-10380 (2006).

Jørgensen BM, Rasmussen HN, Rasmussen UF: Ubiquinone reduction pattern in pigeon heart mitochondria. Identification of three distinct ubiquinone pools. Biochem J 229: 621-629 (1985).

Kaambre T, Chekulayev V, Shevchuk I, KaruVarikmaa M, Timohhina N, et al: Metabolic control analysis of cellular respiration in situ in intraoperational samples of human breast cancer. J Bioenerg Biomembr 44:539-558 (2012).

Kang SY, Gutowsky HS, Hsung JC, Jacobs R, King TE, et al: Nuclear magnetic resonance investigation of the cytochrome oxidase-phospholipid interaction: a new model for boundary lipid. Biochemistry 18:3257-3267 (1979).

Khalifat N, Puff N, Bonneau S, Fournier JB, Angelova MI: Membrane deformation under local pH gradient: mimicking mitochondrial cristae dynamics. Biophys J 95:4924-4933 (2008).

Khalifat N, Fournier JB, Angelova MI, Puff N: Lipid packing variations induced by $\mathrm{pH}$ in cardiolipin-containing bilayers: the driving force for the cristae-like shape instability. Biochim Biophys Acta 1808:2724-2733 (2011).

Kholodenko BN, Westerhoff HV: Metabolic channelling and control of the flux. FEBS Lett 320:71-74 (1993).
Kröger A, Klingenberg M: The kinetics of the redox reactions of ubiquinone related to the electron-transport activity in the respiratory chain. Eur J Biochem 34:358-368 (1973).

Kulawiak B, Höpker J, Gebert M, Guiard B, Wiedemann N, et al: The mitochondrial protein import machinery has multiple connections to the respiratory chain. Biochim Biophys Acta 1827:612-626 (2013).

-Lamantea E, Carrara F, Mariotti C, Morandi L, Tiranti V, et al: A novel nonsense mutation (Q352X) in the mitochondrial cytochrome $b$ gene associated with a combined deficiency of complexes I and III. Neuromuscul Disord 12: 49-52 (2002).

Lange C, Nett JH, Trumpower BL, Hunte C: Specific roles of protein-phospholipid interactions in the yeast cytochrome bcl complex structure. EMBO J 20:6591-6600 (2001).

Lapuente-Brun E, Moreno-Loshuertos R, AcínPeréz R, Latorre-Pellicer A, Colás C, et al: Supercomplex assembly determines electron flux in the mitochondrial electron transport chain. Science 340:1567-1570 (2013).

Lass A: Electron transport-linked ubiquinone-dependent recycling of $\alpha$-tocopherol inhibits autooxidation of mitochondrial membranes. Arch Biochem Biophys 352:229-236 (1998).

Lass A, Sohal RS: Comparisons of coenzyme Q bound to mitochondrial membrane proteins among different mammalian species. Free Radic Biol Med 27:220-226 (1999).

Lee I, Salomon AR, Ficarro S, Mathes I, Lottspeich F, et al: cAMP-dependent tyrosine phosphorylation of subunit I inhibits cytochrome c oxidase activity. J Biol Chem 280:6094-6100 (2005).

Lenaz G: A critical appraisal of the mitochondrial coenzyme Q pool. FEBS Lett 509:151-155 (2001).

Lenaz G, Genova ML: Kinetics of integrated electron transfer in the mitochondrial respiratory chain: random collisions vs. solid state electron channeling. Am J Physiol Cell Physiol 292:C1221-C1239 (2007).

Lenaz G, Genova ML: Structure and organization of mitochondrial respiratory complexes: a new understanding of an old subject. Antioxid Redox Signal 12:961-1008 (2010).

Lenaz G, Genova ML: Supramolecular organisation of the mitochondrial respiratory chain: a new challenge for the mechanism and control of oxidative phosphorylation. Adv Exp Med Biol 748:107-144 (2012).

Lenaz G, Fato R, Di Bernardo S, Jarreta D, Costa $\mathrm{A}$, et al: Localization and mobility of coenzyme Q in lipid bilayers and membranes. Biofactors 9:87-93 (1999).

Lenaz G, Baracca A, Barbero G, Bergamini C, Dalmonte ME, et al: Mitochondrial respiratory chain supercomplex I-III in physiology and pathology. Biochim Biophys Acta 1797:633640 (2010)

Maas MF, Krause F, Dencher NA, Sainsard-Chanet A: Respiratory complexes III and IV are not essential for the assembly/stability of complex I in fungi. J Mol Biol 387:259-269 (2009). 
MacLennan DH, Lenaz G, Szarkowska L: Studies on the mechanism of oxidative phosphorylation. IX. Effect of cytochrome c on energylinked processes. J Biol Chem 241:5251-5259 (1966).

- Maj MC, Raha S, Myint T, Robinson BH: Regulation of NADH/CoQ oxidoreductase: do phosphorylation events affect activity? Protein I 23:25-32 (2004).

-Maranzana E, Barbero G, Falasca AI, Lenaz G, Genova ML: Mitochondrial respiratory supercomplex association limits production of reactive oxygen species from complex I. Antioxid Redox Signal 19:1469-1480 (2013).

-Matthews RT, Yang L, Browne S, Baik M, Beal MF: Coenzyme Q10 administration increases brain mitochondrial concentrations and exerts neuroprotective effects. Proc Natl Acad Sci USA 95:8892-8897 (1998).

-McKenzie M, Lazarou M, Thorburn DR, Ryan MT: Mitochondrial respiratory chain supercomplexes are destabilized in Barth syndrome patients. J Mol Biol 361:462-469 (2006).

-Molyneux SL, Florkowski CM, Richards AM, Lever $\mathrm{M}$, Young JM, et al: Coenzyme Q10; an adjunctive therapy for congestive heart failure? N Z Med J 122:74-79 (2009).

-Moreno Lastres D, Fontanesi F, García-Consuegra I, Martín MA, Arenas J, et al: Mitochondrial complex I plays an essential role in human respirasome assembly. Cell Metab 15: 324-335 (2012).

-Mracek T, Holzerová E, Drahota Z, Kovářová N, Vrbacky M, et al: ROS generation and multiple forms of mammalian mitochondrial glycerol-3-phosphate dehydrogenase Biochim Biophys Acta 1837:98-111 (2014).

-Murphy MP: Mitochondrial thiols in antioxidant protection and redox signaling: distinct roles for glutathionylation and other thiol modifications. Antioxid Redox Signal 16:476-495 (2012).

- Muster B, Kohl W, Wittig I, Strecker V, Joos F, et al: Respiratory chain complexes in dynamic mitochondria display a patchy distribution in life cells. PLoS ONE 5:e11910 (2010).

-Neuwald AF: Barth syndrome may be due to an acyltransferase deficiency. Curr Biol 7:R465466 (1997).

-Osman C, Voelker DR, Langer T: Making heads or tails of phospholipids in mitochondria. J Cell Biol 192:7-16 (2011).

-Ovádi J: Physiological significance of metabolic channelling. J Theor Biol 152:1-22 (1991).

- Panov A, Dikalov S, Shalbuyeva N, Hemendinger $\mathrm{R}$, Greenamyre JT, et al: Species- and tissuespecific relationships between mitochondrial permeability transition and generation of ROS in brain and liver mitochondria of rats and mice. Am J Physiol Cell Physiol 292:C708C718 (2007).

- Papa S, de Rasmo D, Scacco S, Signorile A, Technikova-Dobrova $Z$, et al: Mammalian complex I: a regulable and vulnerable pacemaker in mitochondrial respiratory function. Biochim Biophys Acta 1777:719-728 (2008).
Paradies G, Petrosillo G, Pistolese M, Ruggiero FM: The effect of reactive oxygen species generated from the mitochondrial electron transport chain on the cytochrome $c$ oxidase activity and on the cardiolipin content in bovine heart submitochondrial particles. FEBS Lett 466:323-326 (2000).

Paradies G, Petrosillo G, Pistolese M, Ruggiero FM: Reactive oxygen species affect mitochondrial electron transport complex I activity through oxidative cardiolipin damage. Gene 286:135-141 (2002).

- Paradies G, Petrosillo G, Paradies V, Ruggiero FM: Oxidative stress, mitochondrial bioenergetics, and cardiolipin in aging. Free Radic Biol Med 48:1286-1295 (2010).

-Perales-Clemente E, Bayona-Bafaluy MP, PérezMartos A, Barrientos A, Fernández-Silva P, et al: Restoration of electron transport without proton pumping in mammalian mitochondria. Proc Natl Acad Sci USA 105:1873518739 (2008).

-Petrosillo G, Ruggiero FM, Di Venosa N, Paradies G: Decreased complex III activity in mitochondria isolated from rat heart subjected to ischemia and reperfusion: role of reactive oxygen species and cardiolipin. FASEB J 17: 714-716 (2003).

Pfeiffer K: Cardiolipin stabilizes respiratory chain supercomplexes. J Biol Chem 278:5287352880 (2003)

-Piccoli C, Scrima R, Boffoli D, Capitanio N: Control by cytochrome $\mathrm{c}$ oxidase of the cellular oxidative phosphorylation system depends on the mitochondrial energy state. Biochem J 396:573-583 (2006).

Quarato G, Piccoli C, Scrima R, Capitanio N: Variation of flux control coefficient of cytochrome c oxidase and of the other respiratory chain complexes at different values of protonmotive force occurs by a threshold mechanism. Biochim Biophys Acta 1807:1114-1124 (2011).

Quinzii CM, Hirano M: Coenzyme Q and mitochondrial disease. Dev Disabil Res Revs 16: 183-188 (2010).

Radermacher M, Ruiz T, Clason T, Benjamin S, Brandt $\mathrm{U}$, et al: The three-dimensional structure of complex I from Yarrowia lipolytica: a highly dynamic enzyme. J Struct Biol 154: 269-279 (2006).

Ragan CI, Heron C: The interaction between mitochondrial NADH-ubiquinone oxidoreductase and ubiquinol-cytochrome $c$ oxidoreductase. Evidence for stoicheiometric association. Biochem J 174:783-790 (1978).

- Raha S, Myint AT, Johnstone L, Robinson BH: Control of oxygen free radical formation from mitochondrial complex I: roles for protein kinase $\mathrm{A}$ and pyruvate dehydrogenase kinase. Free Radic Biol Med 32:421-430 (2002).

Ramirez-Aguilar SJ, Keuthe M, Rocha M, Fedyaev VV, Kramp K, et al: The composition of plant mitochondrial supercomplexes changes with oxygen availability. J Biol Chem 286:4304543053 (2011).
Rauchová H, Fato R, Drahota Z, Lenaz G: Steadystate kinetics of reduction of coenzyme Q analogs by glycerol-3-phosphate dehydrogenase in brown adipose tissue mitochondria. Arch Biochem Biophys 344:235-241 (1997).

Rodrigues JV, Gomes CM: Mechanism of superoxide and hydrogen peroxide generation by human electron-transfer flavoprotein and pathological variants. Free Radic Biol Med 53: 12-19 (2012).

Rosca M, Hoppel C: New aspects of impaired mitochondrial function in heart failure. J Bioenerg Biomembr 41:107-112 (2009).

- Rosca M, Vazquez E, Kerner J, Parland W, Chandler M, et al: Cardiac mitochondria in heart failure: decrease in respirasomes and oxidative phosphorylation. Cardiovasc Res 80:3039 (2008).

Rosca M, Minkler P, Hoppel CL: Cardiac mitochondria in heart failure: normal cardiolipin profile and increased threonine phosphorylation of complex IV. Biochim Biophys Acta 1807:1373-1382 (2011).

Rosenfeldt F, Marasco S, Lyon W, Wowk M, Sheeran F, et al: Coenzyme Q10 therapy before cardiac surgery improves mitochondrial function and in vitro contractility of myocardial tissue. J Thorac Cardiovasc Surg 129:2532 (2005).

Rötig A, Appelkvist EL, Geromel V, Chretien D, Kadhom N, et al: Quinone-responsive multiple respiratory-chain dysfunction due to widespread coenzyme Q10 deficiency. Lancet 356:391-395 (2000).

-Schäfer E, Seelert H, Reifschneider NH, Krause F, Dencher NA, et al: Architecture of active mammalian respiratory chain supercomplexes. J Biol Chem 281:15370-15375 (2006).

-Schäfer E, Dencher NA, Vonck J, Parcej DN: Three-dimensional structure of the respiratory chain supercomplex I1III2IV1 from bovine heart mitochondria. Biochemistry 46: 12579-12585 (2007).

Schägger H, Pfeiffer K: Supercomplexes in the respiratory chains of yeast and mammalian mitochondria. EMBO J 19:1777-1783 (2000).

- Schägger $\mathrm{H}$, Pfeiffer $\mathrm{K}$ : The ratio of oxidative phosphorylation complexes $\mathrm{I}-\mathrm{V}$ in bovine heart mitochondria and the composition of respiratory chain supercomplexes. J Biol Chem 276:37861-37867 (2001).

-Schägger H, de Coo R, Bauer MF, Hofmann S, Godinot C, et al: Significance of respirasomes for the assembly/stability of human respiratory chain complex I. J Biol Chem 279:3634936353 (2004).

Schneider H, Lemasters JJ, Hackenbrock CR: Lateral diffusion of ubiquinone during electron transfer in phospholipid- and ubiquinoneenriched mitochondrial membranes. J Biol Chem 257:10789-10793 (1982).

-Sedlák E, Robinson NC: Phospholipase A(2) digestion of cardiolipin bound to bovine cytochrome c oxidase alters both activity and quaternary structure. Biochemistry 38:1496614972 (1999). 
-Seelert H, Dani DN, Dante S, Hauß T, Krause F, et al: From protons to OXPHOS supercomplexes and Alzheimer's disease: structure-dynamics-function relationships of energytransducing membranes. Biochim Biophys Acta 1787:657-671 (2009).

-Skulachev VP: Role of uncoupled and non-coupled oxidations in maintenance of safely low levels of oxygen and its one-electron reductants. Q Rev Biophys 29:169-202 (1996).

-Sousa PM, Silva ST, Hood BL, Charro N, Carita JN: Supramolecular organizations in the aerobic respiratory chain of Escherichia coli. Biochimie 93:418-425 (2011).

Stoner CD: Steady-state kinetics of the overall oxidative phosphorylation reaction in heart mitochondria. Determination of the coupling relationships between the respiratory reactions and miscellaneous observations concerning rate-limiting steps. J Bioenerg Biomembr 16:115-141 (1984)

-Strogolova V, Furness A, Robb-McGrath M, Garlich J, Stuart RA: Rcf1 and Rcf2, members of the hypoxia induced gene 1 protein family, are critical components of the mitochondrial cytochrome bc1-cytochrome coxidase supercomplex. Mol Cell Biol 32:1363-1373 (2012).

Suthammarak W, Yang Y-Y, Morgan PG, Sedensky MM: Complex I function is defective in complex IV-deficient Caenorhabditis elegans. J Biol Chem 284:6425-6435 (2009).

-Suthammarak W, Morgan PG, Sedensky MM: Mutations in mitochondrial complex III uniquely affect complex I in Caenorhabditis elegans. J Biol Chem 285:40724-40731 (2010).
Trouillard M, Meunier B, Rappaport F: Questioning the functional relevance of mitochondrial supercomplexes by time-resolved analysis of the respiratory chain. Proc Natl Acad Sci USA 108:1027-1034 (2011).

Tzagoloff A, Barrientos A, Neupert W, Herrmann JM: Atp10p assists assembly of Atp6p into the F0 unit of the yeast mitochondrial ATPase. J Biol Chem 279:19775-19780 (2004).

van den Brink-van der Laan E, Killian JA, de Kruijff B: Nonbilayer lipids affect peripheral and integral membrane proteins via changes in the lateral pressure profile. Biochim Biophys Acta 1666:275-288 (2004).

van Raam BJ, Sluiter W, de Wit E, Roos D, Verhoeven AJ, et al: Mitochondrial membrane potential in human neutrophils is maintained by complex III activity in the absence of supercomplex organisation. PLoS ONE 3:e2013 (2008).

Vempati U, Han X, Moraes CT: Lack of cytochrome $\mathrm{c}$ in mouse fibroblasts disrupts assembly/stability of respiratory complexes I and IV. J Biol Chem 284:4383-4391 (2008).

Vukotic M, Oeljeklaus S, Wiese S, Vögtle FN, Meisinger C, et al: Rcf1 mediates cytochrome oxidase assembly and respirasome formation, revealing heterogeneity of the enzyme complex. Cell Metab 15:336-347 (2012).

-Wang Y, Mohsen A-W, Mihalik SJ, Goetzman ES, Vockley J: Evidence for physical association of mitochondrial fatty acid oxidation and oxidative phosphorylation complexes. J Biol Chem 285:29834-29841 (2010).
Wenz T, Hielscher R, Hellwig P, Schägger H, Richers S, et al: Role of phospholipids in respiratory cytochrome bc(1) complex catalysis and supercomplex formation. Biochim Biophys Acta 1787:609-616 (2009).

Wittig I, Schägger H: Features and applications of blue-native and clear-native electrophoresis. Proteomics 8:3974-3990 (2008).

Xu J, Seo AY, Vorobyeva DA, Carter CS, Anton $\mathrm{SD}$, et al: Beneficial effects of a Q-ter based nutritional mixture on functional performance, mitochondrial function, and oxidative stress in rats. PLoS ONE 5:e10572 (2010).

-Yang X, Dai G, Li G, Yang ES: Coenzyme Q10 reduces beta-amyloid plaque in an APP/PS1 transgenic mouse model of Alzheimer's disease. J Mol Neurosci 41:110-113 (2010).

-Yu CA, Yu L, King TE: Soluble cytochrome b-c1 complex and the reconstitution of succinatecytochrome c reductase. J Biol Chem 249: 4905-4910 (1974).

Zhang L, Yu L, Yu CA: Generation of superoxide anion by succinate-cytochrome $\mathrm{c}$ reductase from bovine heart mitochondria. J Biol Chem 273:33972-33976 (1998).

Zhang M, Mileykovskaya E, Dowhan W: Gluing the respiratory chain together. Cardiolipin is required for supercomplex formation in the inner mitochondrial membrane. J Biol Chem 277:43553-43556 (2002).

Zhao X, Leon IR, Bak S, Mogensen M, Wrzesinski $\mathrm{K}$, et al: Phosphoproteome analysis of functional mitochondria isolated from resting human muscle reveals extensive phosphorylation of inner membrane protein complexes and enzymes. Mol Cell Proteomics 10:M110.000299 (2011). 